\title{
Exogenous shock models: Analytical characterization and probabilistic construction
}

\author{
Matthias Scherer \\ Technische Universität München, Lehrstuhl für Finanzmathematik, \\ Parkring 11, 85748 Garching, Germany, \\ E-mail: scherer@tum.de \\ Henrik Sloot \\ Technische Universität München, Lehrstuhl für Finanzmathematik, \\ Parkring 11, 85748 Garching, Germany, \\ E-mail: henrik.sloot@tum.de
}

\begin{abstract}
A new characterization for survival functions of multivariate failure-times arising in exogenous shock models with non-negative, continuous, and unbounded shocks is presented. These survival-functions are the product of their ordered and individually transformed arguments. The involved transformations may depend on the specific order of the arguments and must fulfill a monotonicity condition. Conversely, every survival function of that very form can be constructed using an exogenous shock model with independent and non-homogeneous shocks.
\end{abstract}

Keywords: Exogenous shock model; fatal shock model; generalized MarshallOlkin distribution; multivariate survival function 


\section{Introduction}

This work is concerned with the analytical characterization and probabilistic construction of multivariate probability laws of random vectors $\left(\tau_{1}, \ldots, \tau_{\mathrm{d}}\right)$ on $\mathbb{R}_{+}^{\mathrm{d}}$ arising from a fatal-shock construction. The seminal model of this kind was presented in [20]. Marshall and Olkin's main objective was to lift the lack-of-memory property to the d-variate case, an ansatz implying a distinct family of survival functions that can be constructed using a fatalshock model involving $2^{\mathrm{d}}-1$ independent and exponentially distributed shocks. More precisely, the failure time of component $i \in\{1, \ldots, d\}=:[d]$ is defined as

$$
\tau_{i}:=\min \left\{Z_{I}:\{i\} \subseteq I \subseteq[d]\right\}, i \in[d],
$$

where $Z_{\mathrm{I}}, \emptyset \neq \mathrm{I} \subseteq[\mathrm{d}]$, are independent exponentially distributed random variables with rates $\lambda_{\mathrm{I}}, \emptyset \neq \mathrm{I} \subseteq[\mathrm{d}]$.

Taking the eponymous Marshall-Olkin construction Eq. (I) as a starting point, various generalizations are possible? Firstly, the operation 'min' might be altered,see [9, Chp. 4.6] for a general concept for constructing multivariate distributions based on a convolution-closed, infinitely divisible class of univariate distributions, which can be used to construct multivariate normal distributions as well as Marshall-Olkin distributions. Second, the assumption of shocks being independent can be dropped, leading for instance to the class of Archimax copulas, also called scale-mixtures of Marshall-Olkin, which assume an Archimedean dependence for the $Z_{I}$, see [1]]. Third, and this is the path we pursue, shock distributions other than the exponential law can be considered. This has been already considered for the bivariate case, see [3, 12] as well as for the exchangeable d-variate case, see [4, 17]. An interesting result, that was derived in [21], is that the class of distributions, which is characterized by a modified lack-of-memory property, where the generic addition is replaced by a reducible and associative binary operator, is a subgroup of GMO distributions with shocks survival functions of the form $\exp \left\{-\lambda_{\mathrm{I}} \mathrm{H}(\mathrm{t})\right\}$. In any of the above cases (or combinations thereof), the price to pay for the addition flexibility is a reduction in mathematical tractability. Deriving the survival function of a generalized d-variate fatalshock model and analyzing its properties is a challenging task. Beyond that, the inverse membership-testing problem, i.e. deciding if a given survival function admits a shock-model representation, is much harder. Hence, it

${ }^{1}$ The functional equation of the lack-of-memory property is another starting point for generalizations, see 21 . 
is not surprising that the bivariate case was investigated first, see [3, 19], followed by cases where the complexity is reduced by a reduction in the amount of considered shocks, see [4], or via some symmetry assumption, see [19, 22]. In [13], many properties of generalized Marshall-Olkin distributions, e.g. the corresponding copulae and coefficients of tail-dependence, are derived.

The main achievement of the present manuscript is Thm. I. It fully characterizes the class of survival functions arising as a particular instance of a fatal-shock model with independent shocks. This characterization is analytic one the one hand, translating the tedious d-increasingness property to a more convenient monotonicity property, and probabilistic on the other hand, establishing precisely how the $2^{\mathrm{d}}-1$ shock distributions must be selected to ultimately arrive at the model in concern.

Closest to the present work is [22], where it is shown that an exchangeable function $C$ mapping $\mathfrak{u} \in[0,1]^{\mathrm{d}}$ to $[0,1]$, defined via a permutation $\pi \in \mathcal{S}_{\mathrm{d}}$ with $\mathfrak{u}_{\pi(1)} \leqslant \ldots \leqslant \mathfrak{u}_{\pi(\mathrm{d})}$, of the form

$$
\mathrm{C}(\mathfrak{u})=\mathfrak{u}_{\pi(1)} \cdot \delta_{2}\left(\mathfrak{u}_{\pi(2)}\right) \cdot \ldots \cdot \delta_{d}\left(u_{\pi(d)}\right)
$$

is a copula if and only if the functions $\left\{\delta_{2}, \ldots, \delta_{\mathrm{d}}\right\}$ fulfill certain monotonicity conditions. This extends the bivariate case treated in $[3]$. Conversely, all copulas of form Eq. (2) admit a stochastic representation as the survival-copula of an exchangeable exogenous shock model, i.e. the shock distribution is equal for any two shocks $Z_{I}$ and $Z_{J}$ sharing the cardinality of their referencing sets $|I|=|J|$. In our analysis we work with survival functions and restrain ourselves from resorting to copulas, as Sklar's separation, see [25], is not as natural in the case of non-exchangeable shock models as it is for exchangeable ones.

To emphasize the relevance of the present study, let us stress that the Marshall-Olkin distribution, mostly due to its embedded lack-of-memory property, arises like a focal point of many inner-mathematical problems. Beyond that, it has been applied in different fields, see [5, 7, 14], most of the applications having a survival-time interpretation/model. For many real-world applications, however, the assumption of exponential shocks needs to be relaxed, see $[2, \sqrt{10}]$, and the resulting model is of the very form that we classify with Thm. 国. 
2 The Generalized Marshall-Olkin distribution

$$
\mathbb{P}\left(\tau_{1}>t_{1}, \ldots, \tau_{d}>t_{d}\right)=\bar{F}(t)=\exp \left\{-\sum_{\emptyset \neq I \subseteq[d]} \lambda_{I} \max _{i \in I} t_{i}\right\} .
$$

One way of generalizing the Marshall-Olkin distribution is to consider time-dependent shock-intensities $s \mapsto \lambda_{\mathrm{I}}(\mathrm{s})$, i.e.

$$
\mathbb{P}\left(Z_{I}>t\right)=\bar{S}_{I}(t)=\exp \left\{-\int_{0}^{t} \lambda_{I}(s) d s\right\}, \forall t \geqslant 0,
$$

where $s \mapsto \lambda_{I}(s)$ is a non-negative function such that the involved integral is finite for all $t \geqslant 0$. In the following, this concept is slightly extended by solely demanding that that cumulative hazard rates $H_{I}(t):=-\log \bar{S}_{I}(t)$ are strictly positive, non-decreasing, zero in $t=0$, and continuous. Particularly, atoms at infinity are allowed and the class of considered survival functions is

$$
\overline{\mathcal{G}}:=\left\{\bar{S}: \mathbb{R}_{+} \rightarrow(0,1]: \bar{S}(0)=1, \bar{S} \in \mathcal{C}^{(0)}\left(\mathbb{R}_{+}\right), \mathrm{d} \overline{\mathrm{S}} \leqslant 0\right\} .
$$

For a set of survival functions $\overline{\mathrm{S}}_{\mathrm{I}} \in \overline{\mathcal{G}}, \emptyset \neq \mathrm{I} \subseteq[\mathrm{d}]$, with corresponding (cumulative) hazard rate functions $\mathrm{H}_{\mathrm{I}}$, fulfilling the (generalized) marginalfiniteness condition

$$
\prod_{\mathrm{I} \supseteq\{i\}} \overline{\mathrm{S}}_{\mathrm{I}} \in \overline{\mathcal{G}}_{1}:=\left\{\overline{\mathrm{S}} \in \overline{\mathcal{G}}: \lim _{\mathrm{t} \rightarrow \infty} \overline{\mathrm{S}}(\mathrm{t}) \rightarrow 0\right\}, \forall \mathrm{i} \in[\mathrm{d}],
$$

the corresponding survival function of a generalized Marshall-Olkin (GMO) distribution is

$$
\overline{\mathrm{F}}(\mathbf{t})=\prod_{\emptyset \neq \mathrm{I} \subseteq[\mathrm{d}]} \bar{S}_{\mathrm{I}}\left(\max _{i \in \mathrm{I}} \mathrm{t}_{\mathrm{i}}\right)=\exp \left\{-\sum_{\emptyset \neq \mathrm{I} \subseteq[\mathrm{d}]} \mathrm{H}_{\mathrm{I}}\left(\max _{i \in \mathrm{I}} \mathrm{t}_{\mathrm{i}}\right)\right\} .
$$

${ }^{2}$ The interpretation $\lambda_{\mathrm{I}}=0 \Leftrightarrow \mathbb{P}\left(Z_{\mathrm{I}}=\infty\right)=1$ requires the marginal-finiteness condition

$$
\sum_{\mathrm{I} \supseteq\{i\}} \lambda_{\mathrm{I}}>0, \forall i \in[\mathrm{d}],
$$

to make the resulting vector $\left(\tau_{1}, \ldots, \tau_{d}\right)$ well defined. 
Note, that, with the (generalized) marginal-finiteness condition, the function

in Eq. (5) is indeed the survival function of a real, non-negative random vector; this follows if an exogenous shock model with shock-survival-functions $\bar{S}_{\mathrm{I}}, \emptyset \neq \mathrm{I} \subseteq[\mathrm{d}]$ is considered.

The survival function in Eq. (5) has an alternative, more compact, representation: Let $t \geqslant 0$ and $\pi \in \mathcal{S}_{\mathrm{d}}$ be a permutation such that $\mathrm{t}_{\pi(1)} \geqslant \ldots \geqslant$ $t_{\pi(d)}$; then, by reordering the factors appropriately, it follows that

$$
\overline{\mathrm{F}}(\mathbf{t})=\prod_{i=1}^{\mathrm{d}} \mathrm{g}_{i}^{\pi}\left(\mathrm{t}_{\pi(\mathfrak{i})}\right)=\prod_{i=1}^{\mathrm{d}} \tilde{g}^{\pi(\{i, \ldots, \mathrm{d}\}) \pi(i)}\left(\mathrm{t}_{\pi(\mathfrak{i})}\right),
$$

where for $i \in[d]$ and $\pi \in \mathcal{S}_{d}$ as well as $\emptyset \neq \mathrm{I} \subseteq[\mathrm{d}]$ and $\mathrm{m} \in \mathrm{I}$

$$
g_{i}^{\pi}(t):=\prod_{I: \pi(i) \in I \subseteq \pi(\{i, \ldots, d\})} \bar{S}_{I}(t)
$$

and

$$
\tilde{g}^{\mathrm{I}, \mathrm{m}}(\mathrm{t}):=\prod_{J: I \cap J=\{m\}} \overline{\mathrm{S}}_{\mathrm{J}}(\mathrm{t}) .
$$

Furthermore, it follows that the factors $g_{i}^{\pi}$ as well as $\tilde{g}^{\mathrm{I}, \mathrm{m}}$, respectively, are in the class of admissible survival functions $\overline{\mathcal{G}}$ and $g_{1}^{\pi}$ as well as $\tilde{g}^{[\mathrm{d}], m}$, respectively, are in the respective subclass with no atoms at infinity $\overline{\mathcal{G}}_{1}$.

The conclusion from the previous paragraph is, that survival functions of GMO-distributed random vectors are the product of their ordered, and individually transformed arguments, i.e. functions of the form as presented in Eq. (6). The following theorem shows, among other things, that a survival function of this kind implies a stochastic representation as an exogenous shock model 3

Theorem 1. Let $\overline{\mathrm{F}}: \mathbb{R}_{+}^{\mathrm{d}} \rightarrow \mathbb{R}$ be a continuous function having a representation as in $E q$. (6) for an arbitrary family of functions $\left\{g_{i}^{\pi}: i \in[d], \pi \in \mathcal{S}_{\mathrm{d}}\right\}$. If additionally

- $\mathrm{g}_{1}^{\pi} \in \overline{\mathcal{G}}_{1} \forall \pi \in \mathcal{S}_{\mathrm{d}}$ and

- $g_{i}^{\pi}(0)=1 \forall i \in[d], \pi \in \mathcal{S}_{d}$,

then the following statements are equivalent:

${ }^{3}$ For readability, the necessary conditions on the transformations $g_{i}^{\pi}$ are omitted here and the reader is referred to the full statement in Thm. 1 . 
1. $\overline{\mathrm{F}}$ is the survival function of a multivariate random vector $\boldsymbol{\tau} \in \mathbb{R}_{+}^{\mathrm{d}}$.

2. For all $\mathrm{I}_{1}, \mathrm{I}_{2} \subseteq[\mathrm{d}]$ with $\mathrm{I}_{1} \cap \mathrm{I}_{2}=\emptyset$ and $\mathrm{I}_{2} \neq \emptyset$, let $\left\{\pi_{\mathrm{J}}\right\}_{\mathrm{J} \subseteq \mathrm{I}_{2}} \subseteq \mathcal{S}_{\mathrm{d}}$ be a family of permutations on $[\mathrm{d}]$ which fulfills for each $\mathrm{J} \subseteq \mathrm{I}_{2}$ the following conditions

(a) $\pi_{\mathrm{J}}\left(\left\{1, \ldots,\left|\mathrm{I}_{1}\right|\right\}\right)=\mathrm{I}_{1}\left(\right.$ if $\left.\mathrm{I}_{1} \neq \emptyset\right)$,

(b) $\pi_{\mathrm{J}}\left(\left\{\left|\mathrm{I}_{1}\right|+1, \ldots,\left|\mathrm{I}_{1} \cup \mathrm{J}\right|\right\}\right)=\mathrm{J}$, and

(c) $\pi_{\mathrm{J}}\left(\left\{\left|\mathrm{I}_{1} \cup \mathrm{J}\right|+1, \ldots,\left|\mathrm{I}_{1} \cup \mathrm{I}_{2}\right|\right\}\right)=\mathrm{I}_{2} \backslash \mathrm{J}$.

Define for $\mathrm{s} \geqslant \mathrm{t} \geqslant 0$

$$
G_{I_{1}, I_{2}}^{\left\{\pi_{J}\right\}_{J \subseteq I_{2}}}(s, t):=\sum_{J \subseteq I_{2}}(-1)^{|J|} \prod_{j=1}^{|J|} g_{\left|I_{1}\right|+j}^{\pi_{J}}(s) \prod_{j=1}^{\left|I_{2} \backslash J\right|} g_{\left|I_{1} \cup J\right|+j}^{\pi_{J}}(t) .
$$

Then $\mathrm{G}_{\mathrm{I}_{1}, \mathrm{I}_{2} \subseteq \mathrm{I}_{2}}^{\left\{\pi_{\mathrm{j}}\right\}_{\mathrm{I}_{2}}}$ does not depend on the specific family $\left\{\pi_{\mathrm{J}}\right\}_{\mathrm{J} \subseteq \mathrm{I}_{2}}$ chosen; therefore, write $\mathrm{G}_{\mathrm{I}_{1}, \mathrm{I}_{2}}$. Furthermore, $\mathrm{G}_{\mathrm{I}_{1}, \mathrm{I}_{2}}(\mathrm{~s}, \mathrm{t})$ is non-negative and continuous in $\mathrm{s}$ and $\mathrm{t}$.

3. For all $\mathrm{I}_{1}, \mathrm{I}_{2} \subseteq[\mathrm{d}]$ with $\mathrm{I}_{1} \cap \mathrm{I}_{2} \neq \emptyset$ and $\mathrm{I}_{2} \neq \emptyset$ define for $\mathrm{m} \in \mathrm{I}_{2}$

$$
\bar{S}_{\mathrm{I}_{1}, \mathrm{I}_{2}}^{\mathrm{m}}(\mathrm{t}):=\prod_{i=1}^{\left|\mathrm{I}_{2}\right|}\left(\prod_{\substack{\mathrm{J} \subseteq \mathrm{I}_{2} \\|J|=i, \mathrm{i} \in \mathrm{J}}} \tilde{g}^{J \cup \mathrm{I}_{1}, \mathrm{~m}_{(}}(\mathrm{t})\right)^{(-1)^{i-1}}, \mathrm{t} \geqslant 0 .
$$

Then $\overline{\mathrm{S}}_{\mathrm{I}_{1}, \mathrm{I}_{2}}^{\mathrm{m}}$ does not depend on the choice of $\mathrm{m}$, i.e. $\overline{\mathrm{S}}_{\mathrm{I}_{1}, \mathrm{I}_{2}}^{\mathrm{m}} \equiv \overline{\mathrm{S}}_{\mathrm{I}_{1}, \mathrm{I}_{2}}$, and $\overline{\mathrm{S}}_{\mathrm{I}_{1}, \mathrm{I}_{2}} \in \overline{\mathrm{G}}_{\text {. }}$.

4. For all $\emptyset \neq \mathrm{I} \subseteq[\mathrm{d}]$ and $\mathrm{m} \in \mathrm{I}$ define

$$
\bar{S}_{I}^{m}(t):=\prod_{i=1}^{|I|}\left(\prod_{\substack{J \subseteq I \\ J \mid=i, m \in J}} \tilde{g}^{J \cup([d] \backslash I), m}(t)\right)^{(-1)^{i-1}}, t \geqslant 0 .
$$

Then $\overline{\mathrm{S}}_{\mathrm{I}}^{\mathrm{m}}$ does not depend on the choice of $\mathrm{m}$, i.e. $\overline{\mathrm{S}}_{\mathrm{I}}^{\mathrm{m}} \equiv \overline{\mathrm{S}}_{\mathrm{I}}$, and $\overline{\mathrm{S}}_{\mathrm{I}} \in \overline{\mathrm{G}}$.

Due to the length of the required notation and the complexity of the theorem, giving an intuitive interpretation is appropriate before providing 
the proof. Therefore, the following paragraph provides detailed interpretations for the statements in Thm. I. To avoid an overflow of phrases like "let ... be" or "If ... is fulfilled, then ...," it is assumed that all objects are used as stated in the theorem and that statement 1 . is fulfilled.

The first part of statement 2. was added to avoid confusion over the choice of $\left\{\pi_{j}\right\}_{J \subseteq I_{2}}$. However, as a direct consequence of $\bar{F}$ having a welldefined representation as in Eq. (6), it is mathematically redundant. The function $\mathrm{G}_{\mathrm{I}_{1}, \mathrm{I}_{2}}^{\left\{\pi_{\mathrm{J}}\right\}_{\mathrm{I}} \mathrm{I}_{2}}$ in Eq. 8 has the interpretation of

$$
\mathrm{G}_{\mathrm{I}_{1}, \mathrm{I}_{2}}^{\left\{\pi_{\mathrm{J}}\right\}_{\subseteq \mathrm{I}_{2}}}(\mathrm{~s}, \mathrm{t})=\mathbb{P}\left(\tau_{\mathrm{i}} \in[\mathrm{t}, \mathrm{s}) \forall \mathrm{i} \in \mathrm{I}_{2} \mid \tau_{\mathrm{i}}>\mathrm{s} \forall \mathrm{i} \in \mathrm{I}_{1}\right)
$$

As it is well-known, see e.g. [24], a multivariate function $F: \mathbb{R}^{d} \rightarrow[0,1]$ is a distribution function if and only if it fulfills the three conditions of "having" margins, groundedness, and non-negative F-volume for all $\mathbf{d}$-boxes $(\mathbf{a}, \mathbf{b}]$, $\mathbf{a}<\mathbf{b}$. The last property guarantees, that all (d-dimensional) rectangles have a non-negative probability, which can be represented with $F$ using the principle of inclusion and exclusion. Particularly, the property reads

$$
\sum_{c \in x_{i=1}^{d}\left\{a_{i}, b_{i}\right\}}(-1)^{\left|a_{i}=c_{i}\right|} F(c) \geqslant 0 .
$$

Moreover, using the principle of inclusion and exclusion, it follows that a function $\overline{\mathrm{F}}$ is a (multivariate) survival function if the corresponding (hypothetical) distribution function, which is defined by

$$
\mathrm{F}(\boldsymbol{x})=1+\sum_{\emptyset \neq \mathrm{I} \subseteq[\mathrm{d}]}(-1)^{|\mathrm{I}|} \overline{\mathrm{F}}\left(\sum_{i \in \mathrm{I}} x_{i} \vec{e}_{i}\right),
$$

is a proper multivariate distribution function. In that spirit, the second part of statement 2. has the interpretation of an "F-volume"-condition. Due to the specific form of the survival function, however, it suffices that the $\overline{\mathrm{F}}$-volumes of some special sets are non-negative. For the exchangeable case, this aspect was further investigated in [22], where an alternative proof of "statement 1 . $\Leftrightarrow$ statement 2." was shown on the copula-level: Each rectangle with non-increasing lower boundaries admits a partition into so called dboxes of the form $X_{i=1}^{m-1}\left(t_{i}, s_{i}\right] \times(t, s]^{d-m+1}$ such that $t_{1} \geqslant \ldots \geqslant t_{m-1} \geqslant t$ and $t_{m-1} \geqslant s$. The special form of the representation in Eq. (6) allows to expand each $\overline{\mathrm{F}}$-volume of a d-box into the product of the $\overline{\mathrm{F}}$-volume of $X_{i=1}^{m-1}\left(t_{i}, s_{i}\right] \times \mathbb{R}_{+}^{d-m+1}$ and $G_{I_{1}, I_{2}}(s, t)$, where $I_{1}$ and $I_{2}$ are arbitrary sets 
with cardinality $m-1$ and $d-m+1 / 4$ respectively:

$$
\begin{aligned}
& \mathbb{P}\left(\tau_{\pi(i)} \in\left(t_{i}, s_{i}\right] \forall i \in[m-1], \tau_{\pi(i)} \in(t, s] \forall i \geqslant m\right) \\
& =\mathbb{P}\left(\tau_{\pi(i)} \in\left(t_{i}, s_{i}\right] \forall i \in[m-1]\right) \cdot G_{\pi([m-1]),[d] \backslash \pi([m-1])}(s, t) .
\end{aligned}
$$

Hence, the question of non-negative $\overline{\mathrm{F}}$-volume can be reduced inductively to statement 2. For the bivariate case, the remaining sets, which have to be tested for non-negativity, are sketched in Fig. 1 . The last part in statement 2. merely reflects the choice of possible shock-distributions, i.e. the class $\overline{\mathcal{G}}$.

Evidently, the statements 3 . and 4 . are closely linked, as the latter is a special case of the former. The last statement contains the formula, how the survival functions of the original shocks can be retrieved from the multivariate survival function of a GMO distribution. Hence, the implication "statement $4 . \Rightarrow$ statement 1 ." can be paraphrased as:

If the formula in Eq. (10), for retrieving the survival functions of the shocks, yields admissible survival functions of class $\overline{\mathcal{G}}$, then $\overline{\mathrm{F}}$ is the survival function of an ESM with shock survival functions $\overline{\mathrm{S}}_{\mathrm{I}}$.

The interpretation of the third statement is a little bit more involved. Given a d-variate model for an ESM and a resulting random vector $\tau$, an important observation, which follows directly from the construction via the min-operator, is that (multivariate)-margins of $\tau$ have a shock model representation, too. Note, that the survival functions of the shocks, corresponding to the marginal model are different, but can be inferred, from those of the full (d-variate) model. To see this, let $\emptyset \neq \mathrm{K} \subsetneq[\mathrm{d}]$ be a proper subset of $[d]$, preferably with a cardinality bigger than one. Then

$$
\tau_{i}=\min \left\{\min \left\{S_{J}: J \cap K=I\right\}: i \in I \subseteq K\right\}, i \in K .
$$

A calculation, which is very similar to the one used to prove that "statement 4. $\Rightarrow$ statement $1 . "$, yields that

$$
\bar{S}_{\mathrm{I}_{1}, \mathrm{I}_{2}}(\mathrm{t})=\prod_{K \subseteq\{1, \ldots, \mathrm{d}\} \backslash\left(\mathrm{I}_{1} \cup \mathrm{I}_{2}\right)} \bar{S}_{\mathrm{I}_{2} \cup K}(\mathrm{t}),
$$

which is the survival function of $\min \left\{\mathrm{S}_{\mathrm{J}}: \mathrm{J} \cap\left(\mathrm{I}_{1} \cup \mathrm{I}_{2}\right)=\mathrm{I}_{2}\right\}$. Hence, statement 3, requires that statement 4 . is fulfilled for every (theoretical) marginal model.

4This reflects the exchangeability of $\overline{\mathrm{F}}$, which is assumed here. 

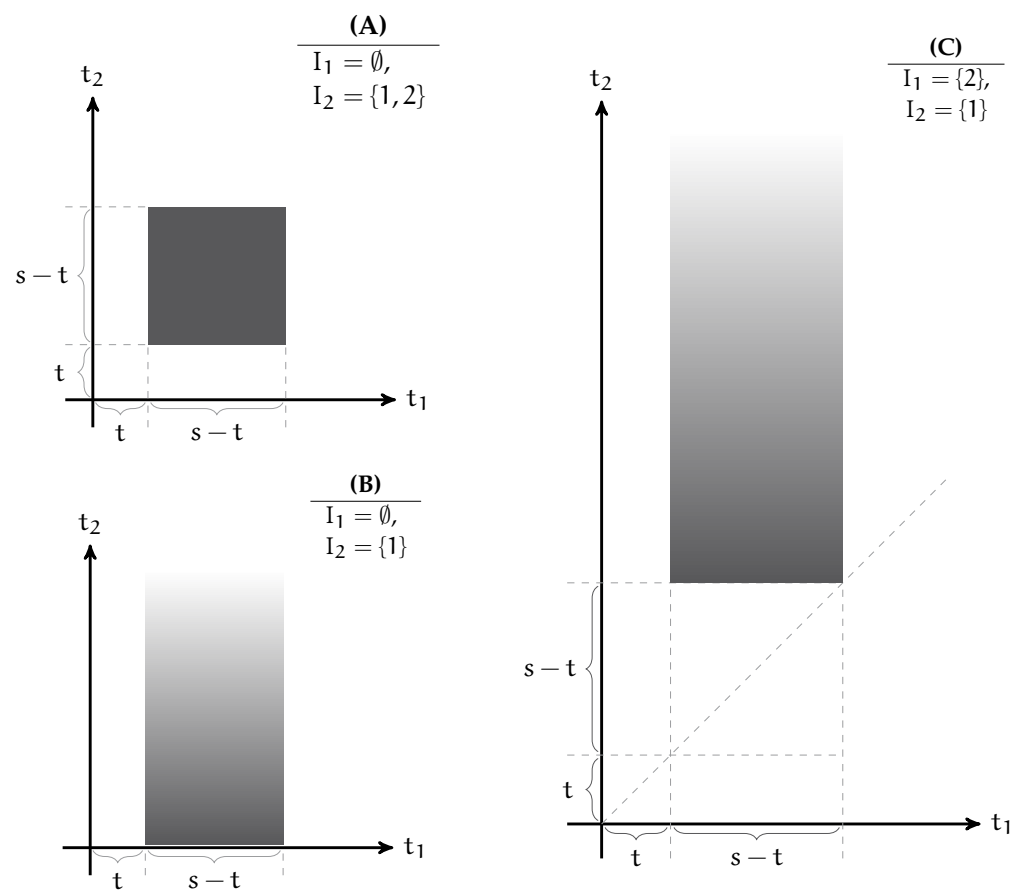

Figure 1: The reduced set of "test-rectangles" for $d=2$, which have to be tested for non-negative " 2 -volume" to verify the validity of a survival function. The three graphs display the three cases, which can be generalized to higher dimensions: (A) Squares, which are split in half by the diagonal, (B) Infinitely expanding rectangles which touch one axis, and (C) Infinitely expanding rectangles which touch the diagonal in one point. 


\section{Proof of the characterization theorem}

The theorem will be proven in four steps. Particularly, it is proved that $3 \Rightarrow 4 \Rightarrow 1 \Rightarrow 2 \Rightarrow 3$.

Remark 1 . Under the assumptions of Thm. I. particularly the representation of $\bar{F}$ in Eq. (6), the expression

$$
g_{i}^{\pi}(t)=\frac{\prod_{j=1}^{i} g_{j}^{\pi}(t)}{\prod_{j=1}^{i-1} g_{j}^{\pi}(t)}
$$

is invariant for different permutations with coinciding images of $[i-1]$ and $i$. If the first statement of the theorem is fulfilled, then $g_{i}^{\pi}$ has the interpretation of a conditional probability, i.e.

$$
g_{i}^{\pi}(t)=\mathbb{P}\left(\tau_{\pi(i)}>t \mid \tau_{\pi([i-1])}>t\right) .
$$

Hence, the function $g_{i}^{\pi}$ only depends on $\pi([i-1])$ and $\pi(i)$ and it is justified to work with $\tilde{g}^{\pi([i]), \pi(i)}$.

Remark 2. Let the assumptions of Thm. $\mathrm{I}$ be fulfilled with $\overline{\mathrm{F}}$ being the survival function of a random vector $\tau$. Then $\tau$ has a stochastic representation as an ESM with shock survival functions $\bar{S}_{\mathrm{I}}$, i.e. if the $\mathrm{Z}_{\mathrm{I}} \sim \overline{\mathrm{S}}_{\mathrm{I}}, \emptyset \neq \mathrm{I} \subseteq[\mathrm{d}]$, are independent shocks and $\tilde{\tau}$ is defined by Eq. $(\mathbb{I})$, then $\tau \stackrel{\mathrm{d}}{=} \tilde{\tau}$.

Proof of $3 \Rightarrow 4$. First observe that 4 . is a special case of 3. , hence 3 . $\Rightarrow 4$. follows directly.

Proof of $4 \Rightarrow 1$. Let 4 . from Thm. I be fulfilled and define for independent random variables $Z_{\mathrm{I}} \sim \bar{S}_{\mathrm{I}}, \emptyset \neq \mathrm{I} \subseteq[\mathrm{d}]$ the random vector $\tau$ by

$$
\tau_{i}:=\min \left\{Z_{I}: i \in I\right\}, i \in[d] .
$$

For $t \geqslant 0$ and $\pi \in \mathcal{S}_{d}$ with $t_{\pi(1)} \geqslant \ldots \geqslant t_{\pi(d)}$, using the independence of the shock variables and reordering the factors, it holds that

$$
\begin{aligned}
\mathbb{P}(\boldsymbol{\tau}>\mathbf{t}) & =\prod_{\emptyset \neq I \subseteq[\mathrm{d}]} \mathbb{P}\left(\mathrm{Z}_{\mathrm{I}}>\max _{i \in \mathrm{I}} \mathrm{t}_{\mathrm{i}}\right) \\
& =\prod_{i=1}^{\mathrm{d}}\left(\prod_{\substack{\mathrm{I} \subseteq \pi(\{i, \mathrm{i}+1, \ldots, \mathrm{d}\}) \\
\pi(i) \in \mathrm{I}}} \mathbb{P}\left(\mathrm{Z}_{\mathrm{I}}>\mathrm{t}_{\pi(\mathrm{i})}\right)\right)
\end{aligned}
$$


For $i \in[d]$ and $\pi(i) \in I \subseteq \pi(\{i, \ldots, d\})$, by assumption, the survival function $\bar{S}_{\mathrm{I}} \equiv \overline{\mathrm{S}}_{\mathrm{I}}^{\pi(\mathrm{i})}$ has a representation as in Eq. $1 \mathrm{IO}$ with $\mathrm{m}=\pi(\mathfrak{i})$ and

$$
\begin{aligned}
& \prod_{\substack{\mathrm{I} \subseteq \pi(\{i, i+1, \ldots, \mathrm{d}\}) \\
\pi(i) \in \mathrm{I}}} \mathbb{P}\left(\mathrm{Z}_{\mathrm{I}}>\mathrm{t}_{\pi(i)}\right) \\
& =\prod_{\substack{I \subseteq \pi(\{i, i+1, \ldots, d\}) \\
\pi(i) \in \mathrm{I}}}\left(\prod_{\substack{J \subseteq I \\
\pi(i) \in J}}\left(\tilde{g}^{J \cup([\mathrm{d}] \backslash \mathrm{I}), \pi(i)}\left(\mathrm{t}_{\pi(i)}\right)\right)^{(-1)^{|J|-1}}\right) .
\end{aligned}
$$

Fix $K \subseteq[\mathrm{d}]$ with $\pi([i]) \subseteq K$; then $i \leqslant|K|=k \leqslant d$ and $1 \leqslant j \leqslant k-i+1$. The expression $\tilde{g}^{K, \pi(i)}\left(t_{\pi(i)}\right)$ with an exponent of $(-1)^{j-1}$ appears $\left(\begin{array}{c}k-i \\ j-1\end{array}\right)$ times, as there are exactly $\left(\begin{array}{c}k-i \\ j-1\end{array}\right)$ possible choices for J with $\pi(i) \in J \subseteq K \backslash \pi([i-1])$. Hence, the overall exponent of the expression $\tilde{g}^{K, \pi(i)}\left(t_{\pi(i)}\right)$ is

$$
\begin{aligned}
& \sum_{j=1}^{k-i+1}(-1)^{j-1}\left(\begin{array}{l}
k-i \\
j-1
\end{array}\right)=\sum_{j=0}^{k-i}(-1)^{j}\left(\begin{array}{c}
k-i \\
j
\end{array}\right) \\
& =(1-1)^{k-i}= \begin{cases}1, & k=i \\
0, & k>i,\end{cases}
\end{aligned}
$$

where the latter expression follows with the binomial formula. Finally, it follows that

$$
\mathbb{P}(\boldsymbol{\tau}>\mathbf{t})=\prod_{i=1}^{\mathrm{d}} \tilde{g}^{\pi([i]), \pi(i)}\left(\mathbf{t}_{\pi(i)}\right)=\prod_{i=1}^{\mathrm{d}} \mathrm{g}_{i}^{\pi}\left(\mathbf{t}_{\pi(i)}\right) .
$$

166

In the following, $I_{1}, I_{2},\left\{\pi_{J}\right\}_{J \subseteq I_{2}}, s$ and $t$ (or a subset of these elements) fulfill the usual conditions if

1. $s>t \geqslant 0$,

2. $\mathrm{I}_{1}, \mathrm{I}_{2} \subseteq[\mathrm{d}]$ with $\mathrm{I}_{1} \cap \mathrm{I}_{2}=\emptyset$ and $\mathrm{I}_{2} \neq \emptyset$,

3. for $\mathrm{J} \subseteq \mathrm{I}_{2}$ one has

(a) $\pi_{\mathrm{J}}\left(\left\{1, \ldots,\left|\mathrm{I}_{1}\right|\right\}\right)=\mathrm{I}_{1}\left(\right.$ if $\left.\mathrm{I}_{1} \neq \emptyset\right)$,

(b) $\pi_{\mathrm{J}}\left(\left\{\left|\mathrm{I}_{1}\right|+1, \ldots,\left|\mathrm{I}_{1} \cup \mathrm{J}\right|\right\}\right)=\mathrm{J}$, 
174

(c) $\pi_{\mathrm{J}}\left(\left\{\left|\mathrm{I}_{1} \cup \mathrm{J}\right|+1, \ldots,\left|\mathrm{I}_{2}\right|\right\}\right)=\mathrm{I}_{2} \backslash \mathrm{J}$.

If only a specific permutation $\pi$ is used, it is assumed that it fulfills this property for $\mathrm{J}=\mathrm{I}_{2}$.

Proof of $1 \Rightarrow 2$. Let 1 . in Thm. $\mathbb{I}$ be fulfilled and let $I_{1}, I_{2},\left\{\pi_{J}\right\}_{J \subseteq I_{2}}, s$ and $t$ fulfill the usual conditions. First assume that for arbitrary $\pi \in \mathcal{S}_{\mathrm{d}}$ and $i \in[d]$ the functions $g_{i}^{\pi}$ are strictly positive on $\mathbb{R}_{+}$. Then

$$
G_{I_{1}, I_{2}}^{\left\{\pi_{J}\right\}_{J} \subseteq I_{2}}(s, t)=\frac{\sum_{J \subseteq I_{2}}(-1)^{|J|} \prod_{j=1}^{\left|I_{1} \cup J\right|} g_{j}^{\pi_{J}}(s) \prod_{j=1}^{\left|I_{2} \backslash J\right|} g_{\left|I_{1} \cup J\right|+j}^{\pi_{J}}(t)}{\prod_{j=1}^{\left|I_{1}\right|} g_{j}^{\pi_{\emptyset}}(s)}
$$

where it is used that by 1 . the diagonal of marginal survival functions of $\tau_{\mathrm{I}_{1}}$ can be represented with every $\pi$ fulfilling $\pi\left(\left\{1, \ldots,\left|I_{1}\right|\right\}\right)=I_{1}$. Particularly, it holds that

$$
\mathbb{P}\left(\tau_{i}>s, i \in I_{1}\right)=\prod_{j=1}^{\left|I_{1}\right|} g_{j}^{\pi_{J_{1}}}(s)=\prod_{j=1}^{\left|I_{1}\right|} g_{j}^{\pi_{J_{2}}}(s), J_{1}, J_{2} \subseteq I_{2}, s \geqslant 0 .
$$

Subsequently, the numerator of Eq. 11 can be rewritten using the principle of inclusion and exclusion as

$$
\begin{aligned}
& \sum_{i=0}^{\left|I_{2}\right|}(-1)^{i} \sum_{J \subseteq I_{2}:|J|=i} \prod_{j=1}^{\left|J \cup I_{1}\right|} g_{j}^{\pi_{J}}(s) \prod_{j=1}^{\left|I_{2} \backslash J\right|} g_{\left|I_{1} \cup J\right|+j}^{\pi_{J}}(t) \\
& =\mathbb{P}\left(A_{\emptyset}^{I_{1}, I_{2}}\right)-\sum_{i=1}^{\left|I_{2}\right|}(-1)^{i+1} \sum_{J \subseteq I_{2}:|J|=i} \mathbb{P}\left(\bigcap_{j \in J} A_{j}^{I_{1}, I_{2}}\right) \\
& =\mathbb{P}\left(A_{\emptyset}^{I_{1}, I_{2}}\right)-\mathbb{P}\left(\bigcup_{i \in I_{2}} A_{i}^{I_{1}, I_{2}}\right)=\mathbb{P}\left(A^{I_{1}, I_{2}}\right),
\end{aligned}
$$

where

$$
\begin{aligned}
& A^{I_{1}, I_{2}}:=\left\{\tau_{i}>s \forall i \in I_{1}, \tau_{i} \in(t, s] \forall i \in I_{2}\right\}, \\
& A_{\emptyset}^{I_{1}, I_{2}}:=\left\{\tau_{i}>s \forall i \in I_{1}, \tau_{i}>t \forall i \in I_{2}\right\}, \text { and } \\
& A_{i}^{I_{1}, I_{2}}:=\left(\bigcap_{j \in I_{1} \cup\{i\}}\left\{\tau_{j}>s\right\}\right) \cap\left(\bigcap_{j \in I_{2} \backslash\{i\}}\left\{\tau_{j}>t\right\}\right), i \in I_{2} .
\end{aligned}
$$


It follows that

$$
\mathrm{G}_{\mathrm{I}_{1}, \mathrm{I}_{2}}^{\left\{\pi_{\mathrm{J}}\right\}_{\mathrm{I}}}(\mathrm{s}, \mathrm{t})=\mathbb{P}\left(\tau_{\mathrm{i}} \in(\mathrm{t}, \mathrm{s}] \forall i \in \mathrm{I}_{2} \mid \tau_{\mathrm{i}}>\mathrm{s} \forall \mathrm{i} \in \mathrm{I}_{1}\right)
$$

and subsequently that $\mathrm{G}_{\mathrm{I}_{1}, \mathrm{I}_{2}}^{\left\{\pi_{\mathrm{I}}\right\}_{\mathrm{I}} \mathrm{I}_{2}}(\mathrm{~s}, \mathrm{t})$ is non-negative and does not depend on the specific choice of $\left\{\pi_{\mathrm{J}}\right\}_{\mathrm{J} \subseteq \mathrm{I}_{2}}$.5

Now, by induction over $i$, the strict positivity, continuity, and nonincreasingness of $g_{i}^{\pi}$ is proven for all $\pi \in \mathcal{S}_{d}$. This implies that $G_{I_{1}, I_{2}}(s, t)$ is continuous in $s$ and $t$. For $i=1$ and $\pi \in \mathcal{S}_{d}$, the assumptions of Thm. $\mathbb{I}$ imply that $g_{1}^{\pi}$ is strictly positive, continuous, and non-increasing. Let the claim be fulfilled for $j<i$, i.e. $g_{j}^{\pi}$ is strictly positive, continuous, and non-increasing for $j \leqslant i-1$ and $\pi \in \mathcal{S}_{d}$.

Right-continuity and left-limits: It is well known, see, e.g., [24, Chp. 6], that copulae are Lipschitz-continuous with constant one. Hence, by exploiting the copula/survival function decomposition, it holds that

$$
\left|\overline{\mathrm{F}}\left(\mathrm{s}_{1}, \ldots, \mathrm{s}_{\mathrm{d}}\right)-\overline{\mathrm{F}}\left(\mathrm{t}_{1}, \ldots, \mathrm{t}_{\mathrm{d}}\right)\right| \leqslant \sum_{i=1}^{\mathrm{d}}\left|\overline{\mathrm{F}}_{\mathrm{i}}\left(\mathrm{s}_{\mathrm{i}}\right)-\overline{\mathrm{F}}_{\mathrm{i}}\left(\mathrm{t}_{\mathrm{i}}\right)\right| \forall \mathbf{t}, \mathbf{s} \geqslant 0
$$

and right-continuity as well as left-limits of $\bar{F}$ are inherited from the margins. For $\pi \in \mathcal{S}_{d}$ the survival function $t \mapsto \mathbb{P}\left(\min _{j \leqslant i} \tau_{\pi(j)}>t\right)$ is rightcontinuous with left-limits and with

$$
g_{i}^{\pi}(t)=\frac{\prod_{j=1}^{i} g_{j}^{\pi}(t)}{\prod_{j=1}^{i-1} g_{j}^{\pi}(t)}=\frac{\mathbb{P}\left(\min _{j \leqslant i} \tau_{\pi(j)}>t\right)}{\prod_{j=1}^{i-1} g_{j}^{\pi}(t)},
$$

right-continuity with left-limits for $\mathrm{g}_{i}^{\pi}$ follows with the induction hypothesis.

Non-increasingness: For $\pi \in \mathcal{S}_{\mathrm{d}}$ and $s \geqslant t \geqslant 0$ define the vector $\boldsymbol{u}(s, t)$ by

$$
u_{\pi(j)}(s, t):= \begin{cases}s, & \forall j<i, \\ t, & j=i, \\ 0, & \forall j>i\end{cases}
$$

5The independence of the specific choice of $\left\{\pi_{\mathrm{J}}\right\}_{\mathrm{J} \subseteq \mathrm{I}_{2}}$ can also be derived without resorting to the probabilistic interpretation by using the assumption that $\overline{\mathrm{F}}$ has a well-defined representation as in Eq. (6). 
Then, by monotonicity of the measure $\mathbb{P}$, one has

$$
\begin{aligned}
& \mathbb{P}(\boldsymbol{\tau}>\mathbf{u}(s, s)) \leqslant \mathbb{P}(\boldsymbol{\tau}>\mathbf{u}(s, t)) \\
& \Leftrightarrow g_{i}^{\pi}(s) \prod_{j=1}^{i-1} g_{j}^{\pi}(s) \leqslant g_{i}^{\pi}(t) \prod_{j=1}^{i-1} g_{j}^{\pi}(s) \\
& \Leftrightarrow g_{i}^{\pi}(s) \leqslant g_{i}^{\pi}(t),
\end{aligned}
$$

where the induction hypothesis, i.e. $g_{j}^{\pi}$ is strictly positive for all $j<i$, is used.

Strict positivity: Assume for $\pi \in \mathcal{S}_{\mathrm{d}}$ that there exists a finite upper bound $s^{\star}$ for strict positivity of $g_{i}^{\pi}$, i.e. $s^{\star}:=\inf \left\{u>0: g_{i}^{\pi}(u)=0\right\}<\infty$, and as $g_{i}^{\pi}$ is right-continuous and non-increasing we have that $g_{i}^{\pi}\left(s^{\star}\right)=0$. For $t<s^{\star}$ we can choose $I_{1}=\pi(\{1, \ldots, i-2\})$ and $I_{2}=\pi(\{i-1, i\})$. Furthermore, let $\tilde{\pi}$ be the permutation which switches the positions of $i-1$ and $i$ in $\pi$, i.e. $\tilde{\pi}=$ $\pi(i-1, i)$. Assume w.l.o.g. that $s^{\star} \leqslant u^{\star}$ for $u^{\star}:=\inf \left\{u>0: g_{i}^{\tilde{\pi}}(u)=0\right\} \in$ $\overline{\mathbb{R}}_{+}$(else switch the roles of $\pi$ and $\tilde{\pi}$ and prove the contradiction for $\tilde{\pi}$ first). Then, with the induction hypothesis it holds that $g_{j}^{\pi}, g_{j}^{\tilde{\pi}}>0 \forall j<i$ and, for $\pi_{\emptyset} \in\{\pi, \tilde{\pi}\}$, that

$$
\begin{aligned}
& 0 \stackrel{\mathrm{IH}}{\leqslant} \mathrm{G}_{\mathrm{I}_{1}, \mathrm{I}_{2}}\left(s^{\star}, t\right)=\prod_{j=i-1}^{i} g_{j}^{\pi_{\emptyset}}(t)-g_{i-1}^{\pi}\left(s^{\star}\right) g_{i}^{\pi}(t) \\
& \quad-g_{i-1}^{\tilde{\pi}}\left(s^{\star}\right) g_{i}^{\tilde{\pi}}(t)+\prod_{j=i-1}^{i} g_{j}^{\pi}\left(s^{\star}\right) \\
& =g_{i-1}^{\pi_{\emptyset}}(t) g_{i}^{\pi_{\emptyset}}(t)-g_{i-1}^{\pi}\left(s^{\star}\right) g_{i}^{\pi}(t)-g_{i-1}^{\tilde{\pi}}\left(s^{\star}\right) g_{i}^{\tilde{\pi}}(t) \\
& =\left\{\begin{array}{l}
\left(g_{i-1}^{\pi}(t)-g_{i-1}^{\pi}\left(s^{\star}\right)\right) g_{i}^{\pi}(t)-g_{i-1}^{\tilde{\pi}}\left(s^{\star}\right) g_{i}^{\tilde{\pi}}(t), \quad \pi_{\emptyset}=\pi \\
\left(g_{i-1}^{\tilde{\pi}}(t)-g_{i-1}^{\tilde{\pi}}\left(s^{\star}\right)\right) g_{i}^{\tilde{\pi}}(t)-g_{i-1}^{\pi}\left(s^{\star}\right) g_{i}^{\pi}(t), \quad \pi_{\emptyset}=\tilde{\pi} .
\end{array}\right.
\end{aligned}
$$

The last expression in Eq. (12) becomes negative if $t$ is sufficiently close to $s^{\star}:$

1. If $u^{\star}>s^{\star}$, choose $\pi_{\emptyset}=\pi$. Then for $t \nearrow s^{\star}$ Eq. (12) approaches $-g_{i-1}^{\tilde{\pi}}\left(s^{\star}\right) g_{i}^{\tilde{\pi}}\left(s^{\star}-\right)$.

As $g_{i-1}^{\tilde{\pi}}\left(s^{\star}\right)>0$ by the induction hypothesis and $g_{i}^{\tilde{\pi}}(t)>0 \forall t<u^{\star}$ with $s^{\star}<u^{\star}$ by the assumption made above it holds that

$$
0 \leqslant-g_{i-1}^{\tilde{\pi}}\left(s^{\star}\right) g_{i}^{\tilde{\pi}}\left(s^{\star}-\right)<0 .
$$


2. If $s^{\star}=u^{\star}$ and $g_{i}^{\pi_{\emptyset}}\left(s^{\star}-\right)>g_{i}^{\pi_{\emptyset}}\left(s^{\star}\right)=0$ for at least one $\pi_{\emptyset} \in\{\pi, \tilde{\pi}\}$, then for $t \nearrow s^{\star}$ Eq. 12 approaches $-g_{i-1}^{\pi_{\emptyset}}\left(s^{\star}\right) g_{i}^{\pi_{\emptyset}}\left(s^{\star}-\right)$.

As $g_{i-1}^{\pi_{\emptyset}}\left(s^{\star}\right)>0$ by the induction hypothesis and $g_{i}^{\pi_{\emptyset}}\left(s^{\star}-\right)>0$ by the assumption made above it holds that

$$
0 \leqslant-g_{i-1}^{\pi_{\emptyset}}\left(s^{\star}\right) g_{i}^{\pi_{\emptyset}}\left(s^{\star}-\right)<0 .
$$

3. Otherwise, as $g_{j}^{\pi_{\emptyset}}$ for $j \in\{i-1$, $i\}$ have left-limits by the induction hypothesis, for every sequence $t_{k} \nearrow s^{\star}$ with $t_{k} \neq s^{\star}$, non-negative sequences $\left\{a_{j, k}^{\pi_{\emptyset}}\right\}_{k \in \mathbb{N}}$ with $a_{j, k}^{\pi_{\emptyset}}\left(s^{\star}-t_{k}\right) \rightarrow 0$ for $k \rightarrow \infty$ can be found s.t.

$$
g_{j}^{\pi_{\emptyset}}\left(t_{k}\right)=g_{j}^{\pi_{\emptyset}}\left(s^{\star}-\right)+a_{j, k}^{\pi_{\emptyset}}\left(s^{\star}-t_{k}\right), j \in\{i-1, i\}, k \in \mathbb{N} .
$$

By the assumption on $s^{\star}$, it holds that $a_{i, k}^{\pi_{\emptyset}}>0$ for all $k \in \mathbb{N}$ and $\pi_{\emptyset} \in\{\pi, \tilde{\pi}\}$. If $s^{\star}=u^{\star}$ and $g_{i}^{\pi_{\emptyset}}\left(s^{\star}-\right)=g_{i}^{\pi_{\emptyset}}\left(s^{\star}\right)=0$ for all $\pi_{\emptyset} \in\{\pi, \tilde{\pi}\}$, it follows from Eq. 12 and (left-)continuity of $g_{i-1}^{\pi_{\emptyset}}$ that

$$
0 \leqslant \begin{cases}a_{i-1, k}^{\pi} a_{i, k}^{\pi}\left(s^{\star}-t_{k}\right)^{2}-g_{i-1}^{\tilde{\pi}}\left(s^{\star}\right) a_{i, k}^{\tilde{\pi}}\left(s^{\star}-t_{k}\right), & \pi_{\emptyset}=\pi \\ a_{i-1, k}^{\tilde{\pi}} a_{i, k}^{\tilde{\pi}}\left(s^{\star}-t_{k}\right)^{2}-g_{i-1}^{\pi}\left(s^{\star}\right) a_{i, k}^{\pi}\left(s^{\star}-t_{k}\right), & \pi_{\emptyset}=\tilde{\pi}\end{cases}
$$

or equivalently

$$
0 \leqslant \begin{cases}a_{i-1, k}^{\pi}\left(s^{\star}-t_{k}\right) \frac{a_{i, k}^{\pi}}{a_{i, k}^{\tilde{\pi}}}-g_{i-1}^{\tilde{\pi}}\left(s^{\star}\right), & \pi_{\emptyset}=\pi \\ a_{i-1, k}^{\tilde{\pi}}\left(s^{\star}-t_{k}\right) \frac{a_{i, k}^{\tilde{\pi}}}{a_{i, k}^{\pi}}-g_{i-1}^{\pi}\left(s^{\star}\right), & \pi_{\emptyset}=\tilde{\pi} .\end{cases}
$$

Now choose $k$ sufficiently large and $\pi_{\emptyset}$ s.t. the fraction appearing in the upper equation is smaller or equal to 1 , then

$$
\begin{aligned}
0 & \leqslant \begin{cases}a_{i-1, k}^{\pi}\left(s^{\star}-t_{k}\right)-g_{i-1}^{\tilde{\pi}}\left(s^{\star}\right), & a_{i, k}^{\pi} \leqslant a_{i, k}^{\tilde{\pi}} \\
a_{i-1, k}^{\tilde{\pi}}\left(s^{\star}-t_{k}\right)-g_{i-1}^{\pi}\left(s^{\star}\right), & a_{i, k}^{\pi}>a_{i, k}^{\tilde{\pi}}\end{cases} \\
& <0,
\end{aligned}
$$

where it is used that the respective first summand converges for $k \rightarrow \infty$ to 0 and the last summand is negative. Hence, a contradiction is found for each case and therefore $g_{i}^{\pi}(t)>0 \forall t \in \mathbb{R}_{+}$. 
Left-continuity: Let $I_{1}$ and $I_{2}$ as well as $\pi, \tilde{\pi}$, and $\pi_{\emptyset}$ be as above. Then, for all $s>t \geqslant 0$ the function

$$
\mathbb{P}\left(\tau_{i} \in(t, s], i \in I_{2} \mid \tau_{i}>s, i \in I_{1}\right)=G_{I_{1}, I_{2}}(s, t)
$$

has left-limits in $t$. Assume that there exists $s^{\dagger} \in \mathbb{R}_{+}^{\times}$with $g_{i}^{\pi}\left(s^{\dagger}-\right)>g_{i}^{\pi}\left(s^{\dagger}\right)$, then

$$
\begin{aligned}
& 0 \stackrel{\mathrm{IH}}{\leqslant} \lim _{\mathrm{t} \nearrow \mathrm{s}^{\dagger}} \mathrm{G}_{\mathrm{I}_{1}, \mathrm{I}_{2}}\left(\mathrm{~s}^{\dagger}, \mathrm{t}\right) \\
& =\lim _{\mathrm{t} \nearrow^{\dagger}}\left(\prod_{j=i-1}^{i} g_{j}^{\pi_{\emptyset}}(\mathrm{t})-g_{i-1}^{\pi}\left(s^{\dagger}\right) g_{i}^{\pi}(\mathrm{t})-g_{i-1}^{\tilde{\pi}}\left(s^{\dagger}\right) g_{i}^{\tilde{\pi}}(\mathrm{t})\right. \\
& \left.+\prod_{j=i-1}^{i} g_{j}^{\pi}\left(s^{\dagger}\right)\right) \\
& \stackrel{\pi_{\emptyset}=\tilde{\pi},(\star)}{=}\left(g_{i}^{\pi}\left(s^{\dagger}\right)-g_{i}^{\pi}\left(s^{\dagger}-\right)\right) g_{i-1}^{\pi}\left(s^{\dagger}\right)<0,
\end{aligned}
$$

where it is used in $(\star)$, that the first and third summand cancel out, when using that $g_{i-1}^{\tilde{\pi}}$ is continuous under the induction hypothesis. This is a contradiction - hence $g_{i}^{\pi}$ is left-continuous.

Remark 3. The induction in the second part of the proof can be performed on the basis of statement 2. (instead of 1.) from Thm. 1 if the parts on right-continuity with left-limits and non-increasingness are replaced by the following lemma (as they rely on the survival function assumption of 1 .). In particular, 2. implies $g_{i}^{\pi} \in \overline{\mathcal{G}}$ for all $i \in[d], \pi \in \mathcal{S}_{d}$.

Lemma 1. Let 2. from Thm. 1 be fulfilled and $\mathrm{g}_{j}^{\pi}$ be right-continuous with leftlimits, non-increasing, and strictly positive for all $j \leqslant i-1$ and $\pi \in \mathcal{S}_{\mathrm{d}}$. Then $\mathrm{g}_{\mathrm{i}}^{\pi}$ is right-continuous with left-limits and non-increasing for all $\pi \in \mathcal{S}_{\mathrm{d}}$.

Proof. Let $\mathrm{I}_{1}, \mathrm{I}_{2}$, and $\pi$ fulfill the usual conditions with $\left|\mathrm{I}_{2}\right|=2$ and $\left|\mathrm{I}_{1}\right|=$ $i-2$ and define $\tilde{\pi}=\pi(i-1, i)$. 
Right-continuity: Let $s+h>s>t \geqslant 0$. As $\mathrm{G}_{\mathrm{I}_{1}, \mathrm{I}_{2}}(\mathrm{~s}, \mathrm{t})$ is right-continuous in $s$ it holds that

$$
\begin{aligned}
0 & =\lim _{h \searrow 0} G_{I_{1}, I_{2}}(s+h, t)-G_{I_{1}, I_{2}}(s, t) \\
& \stackrel{I H}{=} \underbrace{\lim _{h \searrow 0}\left(g_{i}^{\pi}(s+h)-g_{i}^{\pi}(s)\right),}_{\substack{\text { IH } \\
g_{i-1}^{\pi}(s)}}
\end{aligned}
$$

Left-limits: Let $s>s-h>t \geqslant 0$. As $\mathrm{G}_{\mathrm{I}_{1}, \mathrm{I}_{2}}(s, \mathrm{t})$ and $\mathrm{g}_{i-1}^{\pi_{\emptyset}}(s), \pi_{\emptyset} \in\{\pi, \tilde{\pi}\}$ have left-limits in $s$ and $g_{i-1}^{\pi}$ is positive by induction hypothesis it follows that $g_{i}^{\pi}$ has left-limits:

$$
\begin{aligned}
& \lim _{h \searrow 0} g_{i}^{\pi}(s-h) \\
& =\lim _{h \searrow 0}\left(\frac{G_{I_{1}, I_{2}}(s-h, t)-g_{i-1}^{\tilde{\pi}}(t) g_{i}^{\tilde{\pi}}(t)}{g_{i-1}^{\pi}(s-h)}\right. \\
& \left.-\frac{-g_{i-1}^{\pi}(s-h) g_{i}^{\pi}(t)-g_{i-1}^{\tilde{\pi}}(s-h) g_{i}^{\tilde{\pi}}(t)}{g_{i-1}^{\pi}(s-h)}\right) \text {. }
\end{aligned}
$$

Non-increasingness: Now, let $\mathrm{I}_{1}, \mathrm{I}_{2}$, and $\pi$ fulfill the usual conditions with $I_{2}=\{\pi(i)\}$ and $I_{1}=\pi([i-1])$. As $G_{I_{1}, I_{2}}$ is non-negative, it holds for all $s>t \geqslant 0$ that

$$
0 \leqslant G_{I_{1}, I_{2}}(s, t)=g_{i}^{\pi}(t)-g_{i}^{\pi}(s) .
$$

Lemma 2. Assume that statement 2. of Thm. 1 is fulfilled and let $\mathrm{I}_{1}$ and $\mathrm{I}_{2}$ fulfill the usual conditions. Then for each $\mathrm{m} \in \mathrm{I}_{2}, \overline{\mathrm{S}}_{\mathrm{I}_{1}, \mathrm{I}_{2}}^{\mathrm{m}}$ is an $\mathbb{R}_{+}$-valued, positive, and continuous function on $\mathbb{R}_{+}$. Furthermore, $\overline{\mathrm{S}}_{\mathrm{I}_{1}, \mathrm{I}_{2}}^{\mathrm{m}}$ does not depend on $\mathrm{m} \in \mathrm{I}_{2}$, i.e.

$$
\overline{\mathrm{S}}_{\mathrm{I}_{1}, \mathrm{I}_{2}}^{\mathrm{m}_{1}}(\mathrm{t})=\overline{\mathrm{S}}_{\mathrm{I}_{1}, \mathrm{I}_{2}}^{\mathrm{m}_{2}}(\mathrm{t}) \forall \mathrm{t} \geqslant 0, \mathrm{~m}_{1}, \mathrm{~m}_{2} \in \mathrm{I}_{2} \text {. }
$$

Proof. For $\pi \in \mathcal{S}_{\mathrm{d}}$, due to Rmk. 3 and Lem. I it follows that the functions $g_{i}^{\pi}, i=1, \ldots, d$ are positive, continuous functions on $\mathbb{R}_{+}$. Hence $\bar{S}_{\mathrm{I}_{1}, \mathrm{I}_{2}}^{\mathrm{m}}$ is an $\mathbb{R}_{+}$-valued, positive, and continuous function for every $\mathrm{I}_{1}, \mathrm{I}_{2}$ fulfilling the usual conditions with $\mathrm{m} \in \mathrm{I}_{2}$. 
In the following, it is proven, by induction over $\left|\mathrm{I}_{2}\right|$, that Eq. (13) holds and furthermore, that for all $I_{1}$ and $I_{2}$ fulfilling the usual conditions

$$
\prod_{i=1}^{\left|I_{2}\right|} g_{\left|I_{1}\right|+i}^{\tilde{\pi}}(t)=\prod_{i=1}^{\left|I_{2}\right|} g_{\left|I_{1}\right|+i}^{\hat{\pi}}(t) \forall t \geqslant 0
$$

for all $\tilde{\pi}, \hat{\pi} \in \mathcal{S}_{\mathrm{d}}$ fulfilling $\pi\left(\left[\left|\mathrm{I}_{1}\right|\right]\right)=\mathrm{I}_{1}$ and $\pi\left(\left[\left|\mathrm{I}_{1} \cup \mathrm{I}_{2}\right|\right] \backslash\left[\left|\mathrm{I}_{1}\right|\right]\right)=\mathrm{I}_{2}$ for $\pi \in\{\tilde{\pi}, \hat{\pi}\}$. For $\left|\mathrm{I}_{2}\right|=1$ both claims are naturally fulfilled. Let both claims be fulfilled for $\left|I_{2}\right|<p$ and let $I_{1}, I_{2}$ as well as $\pi$ fulfill the usual conditions with $\left|I_{2}\right|=p, m \in I_{2}$ as well as $\pi\left(\left|I_{1}\right|+1\right)=m$, then for $t \geqslant 0$

$$
\begin{aligned}
& \prod_{\emptyset \neq J \subseteq I_{2}} \bar{S}_{I_{1} \cup\left(I_{2} \backslash J\right), J}^{\pi\left(\min _{j \in J} \pi^{-1}(j)\right)}(t) \\
& \stackrel{(\star)}{=} \prod_{i=1}^{\left|I_{2}\right|} \prod_{\substack{J \subseteq \pi\left(\left\{\left|I_{1}\right|+i, \ldots,\left|I_{1} \cup I_{2}\right|\right\}\right) \\
\pi\left(\left|I_{1}\right|+i\right) \in J}} \bar{S}_{I_{1} \cup\left(I_{2} \backslash J\right), J}^{\pi\left(\left|I_{1}\right|+i\right)}(t) \\
& =\prod_{i=1}^{\left|I_{2}\right|} \prod_{\substack{J \subseteq \pi\left(\left\{\left|I_{1}\right|+i, \ldots,\left|I_{1} \cup I_{2}\right|\right\}\right) \\
\pi\left(\left|I_{1}\right|+i\right) \in J}} \\
& \times \prod_{\substack{\mathrm{L} \subseteq J \\
\pi\left(\left|\mathrm{I}_{1}\right|+i\right) \in \mathrm{L}}}\left(\tilde{g}^{\mathrm{L} \cup \mathrm{I}_{1} \cup\left(\mathrm{I}_{2} \backslash J\right), \pi\left(\left|\mathrm{I}_{1}\right|+\mathfrak{i}\right)}(\mathrm{t})\right)^{(-1)^{|\mathrm{L}|-1}},
\end{aligned}
$$

where the factors in $(\star)$ are regrouped in a similar sense as for the alternative representation for the GMO survival function.

Now for $i \in[\mathrm{d}]$ fix $\pi\left(\left\{1, \ldots,\left|\mathrm{I}_{1}\right|+i\right\}\right) \subseteq \mathrm{K} \subseteq \mathrm{I}_{1} \cup \mathrm{I}_{2}$ and define $\mathrm{k}=|\mathrm{K}|$ as well as $1 \leqslant l \leqslant k-\left|I_{1}\right|-i+1$. The expression $\tilde{g}_{k}^{K, \pi\left(\left|I_{1}\right|+i\right)}(t)$ with exponent $(-1)^{l-1}$ appears $\left(\begin{array}{c}k-\left|I_{1}\right|-i \\ l-1\end{array}\right)$ times and the overall exponent for $\tilde{g}_{k}^{K, \pi\left(\left|I_{1}\right|+i\right)}$ is

$$
\sum_{l=1}^{k-i-\left|I_{1}\right|+1}(-1)^{l-1}\left(\begin{array}{cl}
k-i-\left|I_{1}\right| \\
l-1
\end{array}\right)= \begin{cases}1, & k=\left|I_{1}\right|+i \\
0, & \text { else. }\end{cases}
$$

Hence, as it holds for $k=\left|I_{1}\right|+i$ that $K=\pi\left(\left\{1, \ldots,\left|I_{1}\right|+i\right\}\right)$ and

$$
\prod_{\emptyset \neq J \subseteq I_{2}} \bar{S}_{I_{1} \cup\left(I_{2} \backslash J\right), J}^{\pi\left(\min _{j} \in \pi^{-1}(j)\right)}(t)=\prod_{i=1}^{\left|I_{2}\right|} g_{\left|I_{1}\right|+i}^{\pi}(t)
$$


Proof. As in the proof of 4 . to 1 . one can derive analogously for $t \geqslant 0$ and $\pi \in \mathcal{S}_{\mathrm{d}}$ with $\mathrm{t}_{\pi(1)} \geqslant \ldots \geqslant \mathrm{t}_{\pi(\mathrm{d})}$ as well as $\pi\left(\left\{1, \ldots,\left|\mathrm{I}_{1}\right|\right\}\right)=\mathrm{I}_{1}$ and $\pi\left(\left\{\left|\mathrm{I}_{1}\right|+1, \ldots,\left|\mathrm{I}_{1} \cup \mathrm{I}_{2}\right|\right\}\right)=\mathrm{I}_{2}$ that

$$
\mathbb{P}\left(\check{\tau}_{j}>t_{j} \forall j \in I_{2}\right)=\prod_{j=\left|I_{1}\right|+1}^{\left|I_{1} \cup I_{2}\right|} g_{j}^{\pi}\left(t_{\pi(j)}\right)=\prod_{j=1}^{\left|\check{I}_{2}\right|} \check{g}_{j}^{\check{\pi}}\left(\check{\mathfrak{t}}_{\check{\pi}(j)}\right),
$$

where for $\check{\mathrm{I}}_{2}=\left\{1, \ldots,\left|\mathrm{I}_{2}\right|\right\}, \check{\pi} \in \mathcal{S}_{\left|\mathrm{I}_{2}\right|}$ is defined by

$$
\pi\left(\left|I_{1}\right|+j\right)=i_{\check{\pi}(j)} \forall j \in \check{I}_{2}, I_{2}=\left\{i_{1}, \ldots, i_{\left|I_{2}\right|}\right\}
$$

and $\check{g}_{j}^{\check{\pi}}:=g_{\left|I_{1}\right|+j}^{\pi}$ as well as $\check{t}_{\breve{\pi}(j)}:=t_{\pi\left(\left|I_{1}\right|+j\right)}$. Then, it holds for all $0 \leqslant t<s$ that

$$
\mathbb{P}\left(\check{\tau}_{j} \in(\mathrm{t}, \mathrm{s}] \forall j \in \mathrm{I}_{2}\right)=\check{\mathrm{G}}_{\emptyset, \check{I}_{2}}(\mathrm{~s}, \mathrm{t})=\mathrm{G}_{\mathrm{I}_{1}, \mathrm{I}_{2}}(\mathrm{~s}, \mathrm{t}),
$$

${ }_{234}$ where $\check{G}_{\emptyset, \check{I}_{2}}$ corresponds to Eq. $(8)$ w.r.t. $\left\{\check{g}_{j}^{\check{\pi}}\right\}_{j \in \check{I}_{2}, \check{\pi} \in \mathcal{S}_{\left|I_{2}\right|}}$. 
The essence of the previous Lemma is the following: Let $I_{1}$ and $I_{2}$ fulfill the usual conditions, $\mathrm{Z}_{\mathrm{I}} \sim \overline{\mathrm{S}}_{\mathrm{I}} \in \overline{\mathrm{G}}, \emptyset \neq \mathrm{I} \subseteq[\mathrm{d}], \tau$ be defined as in Eq. $[\mathrm{I}]$, and $\check{\tau} \in \mathbb{R}_{+}^{\left|I_{2}\right|}$ be defined by

$$
\check{\tau}_{i}:=\min \left\{\min \left\{Z_{\mathrm{J}}: \mathrm{J} \cap\left(\mathrm{I}_{1} \cup \mathrm{I}_{2}\right)=\mathrm{I}\right\}: i \in \mathrm{I} \subseteq \mathrm{I}_{2}\right\} .
$$

Then

$$
\mathbb{P}\left(\tau_{i} \in(\mathrm{t}, \mathrm{s}] \forall i \in \mathrm{I}_{2} \mid \tau_{i}>s \forall i \in \mathrm{I}_{2}\right)=\mathbb{P}\left(\check{\tau}_{i} \in(\mathrm{t}, \mathrm{s}] \forall i \in \mathrm{I}_{2}\right) \forall \mathrm{s}>\mathrm{t} \geqslant 0 .
$$

Lemma 4. Let $\mathrm{I}_{1}$ and $\mathrm{I}_{2}$ fulfill the usual conditions. Then, for a specific family $\left\{\pi_{J}\right\}_{J \subseteq \mathrm{I}_{2}}$, the function $\mathrm{G}_{\mathrm{I}_{1}, \mathrm{I}_{2}}^{\left\{\pi_{\mathrm{I}}\right\}_{\subseteq}} \mathrm{I}_{2}$ depends on $\mathrm{g}_{\mathrm{i}}^{\pi_{\mathrm{J}}},\left|\mathrm{I}_{1}\right|+1 \leqslant i \leqslant\left|\mathrm{I}_{1} \cup \mathrm{I}_{2}\right|, \mathrm{J} \subseteq \mathrm{I}_{2}$. Therefore, write

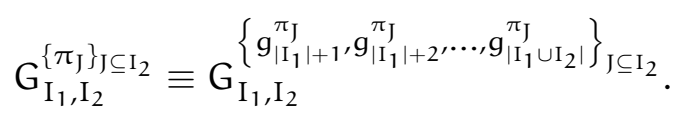

Assume that $\mathrm{g}_{i}^{\pi_{\mathrm{J}}},\left|\mathrm{I}_{1}\right|+1 \leqslant i \leqslant\left|\mathrm{I}_{1} \cup \mathrm{I}_{2}\right|, \mathrm{J} \subseteq \mathrm{I}_{2}$ are positive. Then it holds for all $\mathrm{s} \geqslant \mathrm{t} \geqslant 0$ that

$$
\begin{aligned}
& \left.G_{I_{1}, I_{2}} g_{\mid I_{1}}^{\pi_{I}}, \ldots, g_{I_{1} \cup I_{2} \mid}^{\pi_{J}}\right\}_{J \subseteq I_{2}}(s, t) \\
& =\hat{g}_{\left|I_{1}\right|+1}^{\pi_{\emptyset}}(t) \cdot g_{\left|I_{1}\right|+2}^{\pi_{\emptyset}}(t) \cdot \ldots \cdot g_{\left|I_{1} \cup I_{2}\right|}^{\pi_{\emptyset}}(t) \\
& \times\left(\frac{g_{\left|I_{\emptyset}\right|+1}^{\pi_{\emptyset}}(t)}{\hat{g}_{\left|I_{\emptyset}\right|+1}^{\pi_{\emptyset}}(t)}-\frac{g_{\left|I_{1}\right|+1}^{\pi_{\emptyset}}(s)}{\hat{g}_{\left|I_{1}\right|+1}^{\pi_{\emptyset}}(s)}\right)+\frac{g_{\left|I_{1}\right|+1}^{\pi_{\emptyset}}(s)}{\hat{g}_{\left|I_{1}\right|+1}^{\pi_{\emptyset}}(s)} \\
& \times G_{I_{1}, I_{2}}^{\left\{\hat{g}_{I_{I} \mid+1}^{\pi_{I}}, g_{\left|I_{1}\right|+2}, \ldots, g_{\left|I_{1} \cup I_{2}\right|}^{\pi_{I}}\right\}_{I \subseteq I_{2}}(s, t)}
\end{aligned}
$$

for an arbitrary function $\hat{\mathrm{g}}_{\left|\mathrm{I}_{1}\right|+1}^{\boldsymbol{\pi}_{\emptyset}}$ which is positive on $\mathbb{R}_{+}$, where

$$
\hat{g}_{\left|I_{1}\right|+1}^{\pi_{J}}(s):=\frac{g_{\left|I_{I}\right|+1}^{\pi_{I}}(s)}{g_{\left|I_{\emptyset}\right|+1}^{\pi_{\emptyset}}(s)} \hat{g}_{\left|I_{\emptyset}\right|+1}^{\pi_{\emptyset}}(s), J \subseteq I_{2}, s \geqslant 0,
$$

which are by definition positive functions on $\mathbb{R}_{+}$.

Proof. Every summand corresponding to a non-empty interval $\emptyset \neq \mathrm{J} \subseteq \mathrm{I}_{2}$ contains a term $g_{\left|I_{1}\right|+1}^{\pi_{J}}(s)$. Therefore the result follows by multiplying $G_{I_{1}, I_{2}}$ with $\frac{g_{\left|I_{1}\right|+1}^{\pi_{\emptyset}}(s)}{\hat{g}_{\left|I_{1}\right|+1}^{\pi_{\emptyset}}(s)}$ and its reciprocal, whereas the first summand in Eq. 16 is a correction term for the summand belonging to $\mathrm{J}=\emptyset$. 
Lemma 5. For $k \in \mathbb{N}_{0}, j \geqslant 2$, let the functions $\overline{\mathrm{F}}_{1, k}, \ldots, \overline{\mathrm{F}}_{j, k}:[0, \infty) \rightarrow(0,1]$ as well as $\overline{\mathrm{F}}_{1, k+1}, \ldots, \overline{\mathrm{F}}_{j-1, k+1}:[0, \infty) \rightarrow(0,1]$ be non-increasing with $\overline{\mathrm{F}}_{l, k}=$ $\frac{\overline{\mathrm{F}}_{l-1, k}}{\overline{\mathrm{F}}_{l-1, k+1}}$ for $l \in\{2, \ldots, j\}$. Then it holds that for $\mathrm{s} \geqslant \mathrm{t} \geqslant 0$

$$
0 \leqslant \overline{\mathrm{F}}_{j, k}(\mathrm{t})-\overline{\mathrm{F}}_{j, k}(\mathrm{~s}) \leqslant\left(\prod_{l=1}^{j-1} \frac{1}{\overline{\mathrm{F}}_{l, k+1}(\mathrm{~s})}\right)\left(\overline{\mathrm{F}}_{1, k}(\mathrm{t})-\overline{\mathrm{F}}_{1, k}(\mathrm{~s})\right) .
$$
from

- For $i=1, \ldots, d$ and $\pi \in \mathcal{S}_{d}$, it holds that $g_{i}^{\pi} \in \overline{\mathcal{G}}$.

- For $I_{1}$ and $I_{2}$ fulfilling the usual conditions and $m \in I_{2}$, the function $\bar{S}_{\mathrm{I}_{1}, \mathrm{I}_{2}}^{\mathrm{m}}$ is well-defined as well as positive and continuous. Moreover, it does not depend on the specific $m \in I_{2}$ chosen, hence write $\bar{S}_{I_{1}, I_{2}}$.

It is left to prove that $\bar{S}_{\mathrm{I}_{1}, \mathrm{I}_{2}}$ is non-increasing for all $\mathrm{I}_{1}, \mathrm{I}_{2}$ fulfilling the usual conditions.

The claim is proven by induction over $\left|I_{2}\right|$. For $I_{2}=\{m\}$, let $I_{1}$ and $I_{2}$ fulfill the usual conditions, then $\overline{\mathrm{S}}_{\mathrm{I}_{1}, \mathrm{I}_{2}}=\tilde{\mathrm{g}}^{\mathrm{I}_{1} \cup \mathrm{I}_{2}, \mathrm{~m}} \in \overline{\mathcal{G}}$. Now let $p>1$ and assume that for all $I_{1}$ and $I_{2}$ fulfilling the usual conditions with $\left|I_{2}\right|<p$ it holds that $\bar{S}_{\mathrm{I}_{1}, \mathrm{I}_{2}} \in \overline{\mathcal{G}}$. Let $\mathrm{I}_{1}, \mathrm{I}_{2},\left\{\pi_{\mathrm{J}}\right\}_{\mathrm{J} \subseteq \mathrm{I}_{2}}, s$, and $\mathrm{t}$ fulfill the usual conditions and $\left|I_{2}\right|=p$ and define the function $\hat{g}_{\left|I_{1}\right|+1}^{\pi_{\emptyset}}:=g_{\left|I_{1}\right|+1}^{\pi_{\emptyset}} / \bar{S}_{I_{1}, I_{2}}$, which is continuous and positive. With Lem. 4 it follows that

$$
\begin{aligned}
& 0 \leqslant G_{I_{1}, I_{2}}^{\left\{g_{\left|I_{1}\right|+1}^{\pi_{I}}, g_{\left|I_{1}\right|+2}^{\pi_{I}}, \ldots, g_{\left|I_{1} \cup I_{2}\right|}^{\pi_{I}}\right\}_{J \in I_{2}}(s, t)} \\
& =\hat{g}_{\left|\mathrm{I}_{1}\right|+1}^{\pi_{\emptyset}}(t) g_{\left|\mathrm{I}_{1}\right|+2}^{\pi_{\emptyset}}(t) \cdot \ldots \cdot g_{\left|\mathrm{I}_{1} \cup \mathrm{I}_{2}\right|}^{\pi_{\emptyset}}(t) \\
& \times\left(\bar{S}_{\mathrm{I}_{1}, \mathrm{I}_{2}}(\mathrm{t})-\overline{\mathrm{S}}_{\mathrm{I}_{1}, \mathrm{I}_{2}}(\mathrm{~s})\right)+\overline{\mathrm{S}}_{\mathrm{I}_{1}, \mathrm{I}_{2}}(\mathrm{~s}) \\
& \times G_{I_{1}, I_{2}}^{\left\{\hat{g}_{\left|I_{1}\right|+1}^{\pi_{I}}, g_{\left|I_{1}\right|+2}^{\pi_{J}}, \ldots, g_{\left|I_{1} \cup I_{2}\right|}^{\pi_{J}}\right\}_{J \in I_{2}}}(s, t),
\end{aligned}
$$

where $\hat{g}_{\left|I_{1}\right|+1}^{\pi_{J}}:=g_{|I|+1}^{\pi_{J}} / \bar{S}_{I_{1}, I_{2}}$ for $J \subseteq I_{2}$.

In light of Lem. 3 , it makes sense to derive an exogenous shock model

$$
\left\{\hat{g}_{\left|I_{1}\right|+1}^{\pi_{J}}, g_{\left|I_{1}\right|+2}^{\pi_{J}}, \ldots, g_{\left|I_{1} \cup I_{2}\right|}^{\pi_{J}}\right\}_{J \in I_{2}} .
$$


Hence one has to check, that for $\emptyset \neq \mathrm{J} \subseteq \mathrm{I}_{2}$ if $\overline{\hat{S}}_{\mathrm{I}_{1} \cup \mathrm{I}_{2} \backslash \mathrm{J}, \mathrm{J}} \in \overline{\mathcal{G}}$. Note that

$$
\overline{\hat{S}}_{\mathrm{I}_{1} \cup \mathrm{I}_{2} \backslash J, J}= \begin{cases}\overline{\mathrm{S}}_{\mathrm{I}_{1} \cup \mathrm{I}_{2} \backslash \mathrm{J}, \mathrm{J}}, & \emptyset \neq \mathrm{J} \subsetneq \mathrm{I}_{2} \\ 1, & \mathrm{~J}=\mathrm{I}_{2} .\end{cases}
$$

As $\overline{\mathrm{S}}_{\mathrm{I}_{1} \cup \mathrm{I}_{2} \backslash \mathrm{J}, \mathrm{J}} \in \overline{\mathcal{G}}$ by the induction step for $\emptyset \neq \mathrm{J} \subsetneq \mathrm{I}_{2}$ and $\overline{\hat{S}}_{\mathrm{I}_{1}, \mathrm{I}_{2}} \equiv 1 \in \overline{\mathcal{G}}$, Lem. 3 can be used. Write for $s>t \geqslant 0$

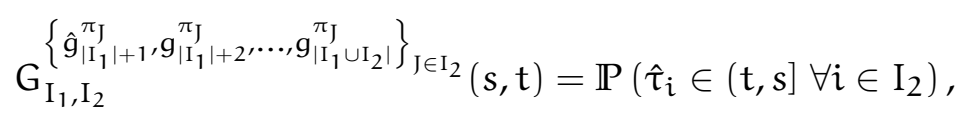

where

$$
\hat{\tau}_{i}:=\min \left\{\hat{Z}_{I}: i \in I \subseteq I_{2}\right\}, i \in I_{2}
$$

with independent $\hat{Z}_{\mathrm{I}} \sim \hat{\mathrm{H}}_{\mathrm{I}_{1} \cup \mathrm{I}_{2} \backslash \mathrm{I}, \mathrm{I}}$ for $\emptyset \neq \mathrm{I} \subseteq \mathrm{I}_{2}$. Let $s>\mathrm{t} \geqslant 0$ and define

$$
\hat{A}^{\mathrm{I}_{1}, \mathrm{I}_{2}}:=\left\{\hat{\tau}_{i} \in(\mathrm{t}, \mathrm{s}] \forall i \in \mathrm{I}_{2}\right\} .
$$

Since $\hat{Z}_{\mathrm{I}_{2}}=\infty$, there are at least two different sets $\emptyset \neq \mathrm{I}, \mathrm{J} \subsetneq \mathrm{I}_{2}$ for which the respective shocks $\hat{Z}_{I}, \hat{Z}_{\text {J }}$ are minimal for one of their components. Moreover, this implies

$$
\hat{A}^{\mathrm{I}_{1}, \mathrm{I}_{2}} \subseteq \bigcup_{\emptyset \neq \mathrm{I}, J \subsetneq \mathrm{I}_{2}: \mathrm{I} \neq \mathrm{J}}\left\{\mathrm{t}<\hat{\mathrm{Z}}_{\mathrm{I}}, \hat{\mathrm{Z}}_{\mathrm{J}} \leqslant s\right\}
$$

From the sub-additivity of the probability measure $\mathbb{P}$, it follows that

$$
\begin{aligned}
\mathbb{P}\left(\hat{A}^{I_{1}, I_{2}}\right) & =G_{I_{1}, I_{2}}^{\left\{\hat{g}_{\left|I_{I}\right|+1}^{\pi_{I}}, g_{\left|I_{1}\right|+2}^{\pi_{J}}, \ldots, g_{\left|I_{1} \cup I_{2}\right|}^{\pi_{J}}\right\}_{J \in I_{2}}(s, t)} \\
& \leqslant \sum_{\substack{\emptyset \neq I_{I J \subsetneq} \subsetneq I_{2} \\
I \neq J}} \mathbb{P}\left(t<\hat{Z}_{I}, \hat{Z}_{J} \leqslant s\right) \\
& \leqslant\left(\begin{array}{c}
2^{\left|I_{2}\right|}-2 \\
2
\end{array}\right) \max _{\emptyset \neq I \subsetneq I_{2}}\left(\bar{S}_{I_{1} \cup I_{2} \backslash I, I}(t)-\bar{S}_{I_{1} \cup I_{2} \backslash I, I}(s)\right)^{2},
\end{aligned}
$$

where we used that for $\emptyset \neq \mathrm{I} \subsetneq \mathrm{I}_{2}$

$$
\mathbb{P}\left(t<\hat{Z}_{I} \leqslant s\right)=\bar{S}_{I_{1} \cup I_{2} \backslash I, I}(t)-\bar{S}_{I_{1} \cup I_{2} \backslash I, I}(s) .
$$


Note that for $\emptyset \neq J \subseteq I \subsetneq I_{2}$ and $m, n \in J, m \neq n$

$$
\begin{aligned}
& \bar{S}_{I_{1} \cup\left(I_{2} \backslash I\right), J}(t)=\bar{S}_{I_{1} \cup\left(I_{2} \backslash I\right), J}^{m}(t) \\
& =\prod_{\substack{\phi \neq \mathrm{L} \subseteq \mathrm{J} \\
\mathrm{m} \in \mathrm{L}}}\left(\tilde{g}^{\mathrm{L} \cup \mathrm{I}_{1} \cup\left(\mathrm{I}_{2} \backslash \mathrm{I}\right), \mathrm{m}}(\mathrm{t})\right)^{(-1)^{|\mathrm{L}|-1}}
\end{aligned}
$$

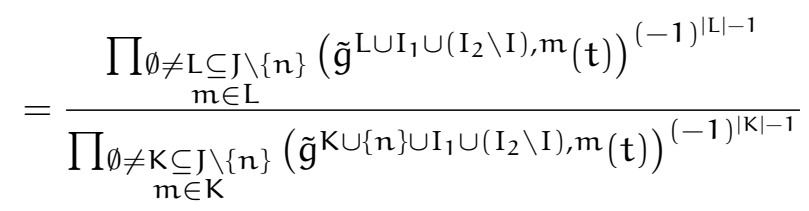

$$
\begin{aligned}
& =\frac{\bar{S}_{I_{1} \cup\left(I_{2} \backslash I\right), J \backslash\{n\}}^{m}(t)}{\bar{S}_{I_{1} \cup\left(I_{2} \backslash I\right) \cup\{n\}, J \backslash\{n\}}(t)}=\frac{\bar{S}_{I_{1} \cup\left(I_{2} \backslash I\right), J \backslash\{n\}}(t)}{\bar{S}_{I_{1} \cup\left(I_{2} \backslash I\right) \cup\{n\}, J \backslash\{n\}}(t)} .
\end{aligned}
$$

Writing $\mathrm{b}:=\left(\begin{array}{c}2^{\left|\mathrm{I}_{2}\right|}-2 \\ 2\end{array}\right)$ and using Lem. 5 for ascending sequences $\emptyset \neq \mathrm{J}_{1} \subsetneq$ $\ldots \subsetneq \mathrm{J}_{|\mathrm{I}|}=\mathrm{I} \subseteq \mathrm{I}_{2}$ with $\left|\mathrm{J}_{\mathrm{I}}\right|=|\mathrm{I}|$ as well as

1. $\overline{\mathrm{F}}_{\left|\mathrm{J}_{l} l,\right| \mathrm{I}_{1} \cup\left(\mathrm{I}_{2} \backslash \mathrm{I}\right) \mid} \equiv \overline{\mathrm{S}}_{\mathrm{I}_{1} \cup\left(\mathrm{I}_{2} \backslash \mathrm{I}\right), \mathrm{J}_{l}}$ for $l \in[|\mathrm{I}|]$ and

2. $\overline{\mathrm{F}}_{\left|\mathrm{J}_{l} l,\right| \mathrm{I}_{1} \cup\left(\mathrm{I}_{2} \backslash \mathrm{I}\right) \cup\left(\mathrm{J}_{l+1} \backslash \mathrm{J}_{l}\right) \mid} \equiv \overline{\mathrm{S}}_{\mathrm{I}_{1} \cup\left(\mathrm{I}_{2} \backslash \mathrm{I}\right) \cup\left(\mathrm{J}_{l+1} \backslash \mathrm{J}_{l}\right), \mathrm{J}_{l}}$ for $l \in[|\mathrm{I}|-1]$

it follows that

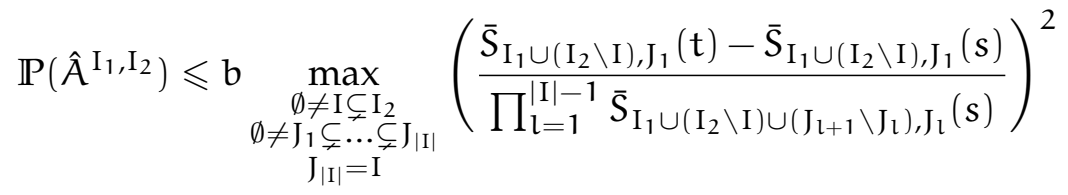

$$
\begin{aligned}
& =\mathrm{b} \max _{\substack{\phi \neq I \subsetneq I_{2} \\
\emptyset \neq \mathrm{J}_{1} \subsetneq \ldots \subsetneq \mathrm{J}_{\mid \mathrm{II}}=\mathrm{I} \\
\mathrm{J}_{1}=\{\mathrm{m}\}}}\left(\frac{\tilde{g}^{\mathrm{I}_{1} \cup\left(\mathrm{I}_{2} \backslash \mathrm{I}\right) \cup \mathrm{J}_{1}, \mathrm{~m}}(\mathrm{t})-\tilde{\mathrm{g}}^{\mathrm{I}_{1} \cup\left(\mathrm{I}_{2} \backslash \mathrm{I}\right) \cup \mathrm{J}_{1}, \mathrm{~m}}(\mathrm{~s})}{\prod_{l=1}^{|\mathrm{I}|-1} \bar{S}_{\mathrm{I}_{1} \cup\left(\mathrm{I}_{2} \backslash \mathrm{I}\right) \cup\left(\mathrm{J}_{\mathrm{l}+1} \backslash \mathrm{J}_{\mathrm{l}}\right) \mathrm{J}_{\mathrm{l}}}(\mathrm{s})}\right)^{2} .
\end{aligned}
$$

Now let $\emptyset \neq \mathrm{I} \subsetneq \mathrm{I}_{2}, \mathrm{k}=\left|\mathrm{I}_{1} \cup\left(\mathrm{I}_{2} \backslash \mathrm{I}\right)\right|, \mathrm{J}_{1}=\{\mathrm{m}\}$ and $\pi \in \mathcal{S}_{\mathrm{d}}$ be a permutation fulfilling $\pi(\{1, \ldots, k\})=I_{1} \cup\left(I_{2} \backslash I\right), \pi(k+1)=m$. Denote with $\tilde{\pi}$ the permutation, which switches the positions of $m$ and $\pi(k)$, i.e. $\tilde{\pi}=\pi(k, k+1)$. Then

$$
\begin{aligned}
0 & \leqslant G_{I_{1} \cup\left(I_{2} \backslash I\right) \backslash\{\pi(k)\},\{m, \pi(k)\}}(s, t) \\
& =\prod_{j=0}^{1} g_{k+j}^{\tilde{\pi}}(t)-g_{k}^{\pi}(s) g_{k+1}^{\pi}(t)-g_{k}^{\tilde{\pi}}(s) g_{k+1}^{\tilde{\pi}}(t)+\prod_{j=0}^{1} g_{k+j}^{\pi}(s) \\
& =g_{k+1}^{\tilde{\pi}}(t)\left(g_{k}^{\tilde{\pi}}(t)-g_{k}^{\tilde{\pi}}(s)\right)-g_{k}^{\pi}(s)\left(g_{k+1}^{\pi}(t)-g_{k+1}^{\pi}(s)\right),
\end{aligned}
$$


which is equivalent to

$$
g_{k+1}^{\pi}(t)-g_{k+1}^{\pi}(s) \leqslant \frac{g_{k+1}^{\tilde{\pi}}(t)}{g_{k}^{\pi}(s)}\left(g_{k}^{\tilde{\pi}}(t)-g_{k}^{\tilde{\pi}}(s)\right) .
$$

This yields inductively the following inequality

$$
\begin{aligned}
g_{k+1}^{\pi}(t)-g_{k+1}^{\pi}(s) \leqslant & \prod_{l=1}^{k} \frac{\tilde{g}^{\pi(\{1, \ldots, l\}) \cup\{m\}, m}(t)}{\tilde{g}^{\pi(\{1, \ldots, l\}), \pi(l)}(s)} \\
& \times\left(\tilde{g}^{\{m\}, m}(t)-\tilde{g}^{\{m\}, m}(s)\right) .
\end{aligned}
$$

Subsequently,

$$
\mathbb{P}\left(\hat{A}^{I_{1}, I_{2}}\right) \leqslant b p_{I_{1}, I_{2}}(s, t) q_{I_{2}}(s, t)
$$

with

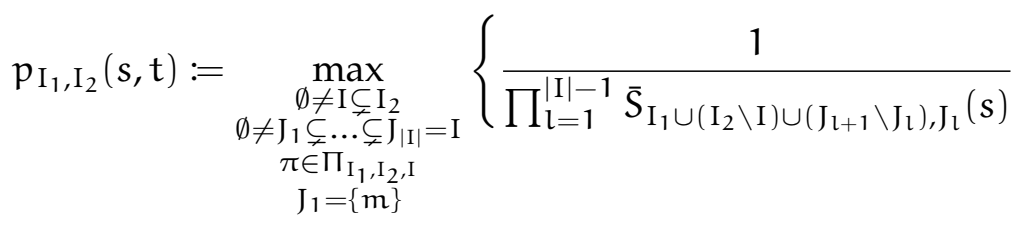

$$
\begin{aligned}
& \left.\times \prod_{l=1}^{\left|\mathrm{I}_{1} \cup\left(\mathrm{I}_{2} \backslash \mathrm{I}\right)\right|} \frac{\tilde{\mathrm{g}}^{\pi(\{1, \ldots, l\}) \cup\{\mathrm{m}\}, \mathrm{m}(\mathrm{t})}}{\tilde{\mathrm{g}}^{\pi(\{1, \ldots, l\}), \pi(\mathrm{l})}(\mathrm{s})}\right\}^{2},
\end{aligned}
$$

where $\Pi_{I_{1}, I_{2}, I}$ is the set of permutations fulfilling the conditions stated above and

$$
\mathrm{q}_{\mathrm{I}_{2}}(\mathrm{~s}, \mathrm{t}):=\max _{\mathrm{m} \in \mathrm{I}_{2}}\left\{\tilde{g}^{\{\mathrm{m}\}, \mathrm{m}}(\mathrm{t})-\tilde{\mathrm{g}}^{\{\mathrm{m}\}, \mathrm{m}}(\mathrm{s})\right\}^{2} .
$$

For $s_{0} \geqslant s>t \geqslant t_{0} \geqslant 0$, the non-increasingness of the functions $\bar{S}_{I_{1} \cup\left(I_{2} \backslash I\right) \cup\left(J_{l+1} \backslash J_{l}\right), J_{l}}(s), \tilde{g}^{\pi(\{[l]\}) \cup\{m\}, m}(t)$, and $\tilde{g}^{\pi(\{[l]\}), \pi(l)}(s)$ implies

$$
p_{I_{1}, I_{2}}(s, t) \leqslant p_{I_{1}, I_{2}}\left(s_{0}, t_{0}\right) \forall t<s, \text { for } t, s \in\left[t_{0}, s_{0}\right] \text {. }
$$

Define for $s \geqslant t \geqslant 0$

$$
\mu_{I_{2}}(s, t)=\sum_{m \in I_{2}} \tilde{g}^{\{m\}, m}(t)-\tilde{g}^{\{m\}, m}(s) .
$$

As $\tilde{g}^{\{m\}, m}, m \in I_{2}$ are non-negative and non-increasing and $\mathrm{q}_{\mathrm{I}_{2}}(\mathrm{~s}, \mathrm{t}) \geqslant 0$ all summands are non-negative and

$$
\mu_{I_{2}}(s, t) \geqslant \sqrt{q_{I_{2}}(s, t)} \geqslant 0, s \geqslant t \geqslant 0
$$


Hence

$$
\begin{aligned}
0 & \leqslant G_{I_{1}, I_{2}}\left\{\hat{g}_{\left|I_{1}\right|+1}^{\pi_{J}}, g_{\left|I_{1}\right|+2}^{\pi_{I}}, \ldots, g_{\left|I_{1} \cup I_{2}\right|}^{\pi_{I}}\right\}_{J \in I_{2}}(s, t) \\
& \leqslant b p_{I_{1}, I_{2}}\left(s_{0}, t_{0}\right) q_{I_{2}}(s, t) \\
& \leqslant b p_{I_{1}, I_{2}}\left(s_{0}, t_{0}\right) \mu_{I_{2}}\left(s_{0}, t_{0}\right)^{2} \forall t, s \in\left[t_{0}, s_{0}\right], t<s .
\end{aligned}
$$

Now, the proof proceeds analogously as for copulas in the exchangeable case [see 17, pp. 1296 sq.] or bivariate exchangeable case [see 3, p. 67].

The function $\bar{S}_{\mathrm{I}_{1}, \mathrm{I}_{2}}$ splits in positive and negative powers in the product terms and

$$
\begin{aligned}
& \bar{S}_{\mathrm{I}_{1}, \mathrm{I}_{2}}(\mathrm{t})=\prod_{i=1}^{\left|\mathrm{I}_{2}\right|}\left(\prod_{\substack{\mathrm{J} \subseteq \mathrm{I}_{2} \\
|J|=i, \mathrm{~m} \in \mathrm{J}}} \tilde{\mathrm{g}}^{\mathrm{J} \cup \mathrm{I}_{1}, \mathrm{~m}}(\mathrm{t})\right)^{(-1)^{\mathrm{i}-1}}
\end{aligned}
$$

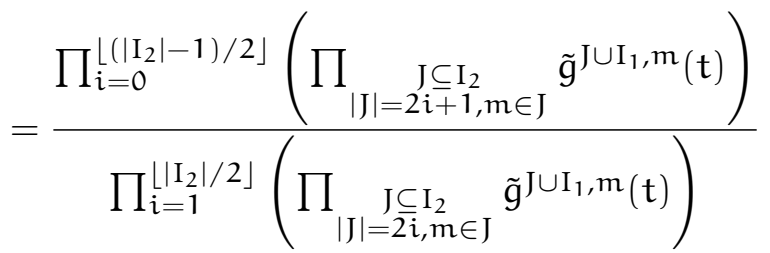

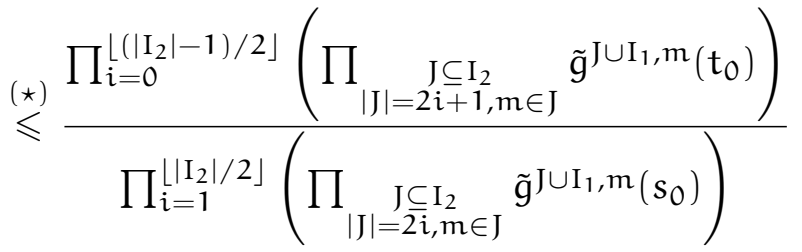

$$
\begin{aligned}
& =: p_{\max }^{I_{1}, I_{2}}\left(s_{0}, t_{0}\right),
\end{aligned}
$$

where the monotonicity of $\tilde{g}^{\mathrm{I}, \mathrm{m}}$ is used in $(\star)$. Assume that $\overline{\mathrm{S}}_{\mathrm{I}_{1}, \mathrm{I}_{2}}$ is not non-increasing, i.e. there exists $s_{0}>t_{0} \geqslant 0$ s.t. $\bar{S}_{I_{1}, I_{2}}\left(s_{0}\right)>\bar{S}_{I_{1}, I_{2}}\left(t_{0}\right)$.

Case $q_{I_{1}}\left(s_{0}, t_{0}\right)=0$ : From Eq. (17) we get

$$
\begin{aligned}
& \left.0 \leqslant G_{I_{1}, I_{2}}^{\left\{g_{\left|I_{J}\right|+1}^{\pi_{I}}, g_{\left|I_{1}\right|+2}^{\pi_{J}}, \ldots, g_{\left|I_{1} \cup I_{2}\right|}^{\pi_{J}}\right.}\right\}_{J \in I_{2}}\left(s_{0}, t_{0}\right) \\
& =\underbrace{\hat{g}_{\left|I_{1}\right|+1}^{\pi_{\emptyset}}\left(t_{0}\right) g_{\left|I_{1}\right|+2}^{\pi_{\emptyset}}\left(t_{0}\right) \ldots g_{\left|I_{1} \cup I_{2}\right|}^{\pi_{\emptyset}}\left(t_{0}\right)}_{>0} \underbrace{\left(\bar{S}_{I_{1}, I_{2}}\left(t_{0}\right)-\bar{S}_{I_{1}, I_{2}}\left(s_{0}\right)\right)}_{<0} \\
& <0
\end{aligned}
$$

which is a contradiction. 
Case $q_{I_{1}}\left(s_{0}, t_{0}\right)>0$ : Let

$$
a\left(s_{0}, t_{0}\right):=\frac{\bar{S}_{I_{1}, I_{2}}\left(s_{0}\right)-\bar{S}_{I_{1}, I_{2}}\left(t_{0}\right)}{\mu_{I_{2}}\left(s_{0}, t_{0}\right)}>0
$$

then we can write

$$
\bar{S}_{I_{1}, I_{2}}\left(t_{0}\right)-\bar{S}_{I_{1}, I_{2}}\left(s_{0}\right)=-a\left(s_{0}, t_{0}\right) \mu_{I_{2}}\left(s_{0}, t_{0}\right)
$$

For all $k \geqslant 1$, one can find $s_{k}, t_{k} \in\left[t_{0}, s_{0}\right]$ with $s_{k}>t_{k}$ and

$$
\mu_{I_{2}}\left(s_{k}, t_{k}\right)=\frac{\mu_{I_{2}}\left(s_{0}, t_{0}\right)}{k}
$$

as well as

$$
\bar{S}_{I_{1}, I_{2}}\left(t_{k}\right)-\bar{S}_{I_{1}, I_{2}}\left(s_{k}\right) \leqslant-a\left(s_{0}, t_{0}\right) \mu_{I_{2}}\left(s_{s}, t_{k}\right)
$$

This can be seen by setting $\mathrm{t}^{(0, k)}:=\mathrm{t}_{0}, \mathrm{t}^{(\mathrm{k}, \mathrm{k})}:=\mathrm{s}_{0}$, and

$$
\mathrm{t}^{(j, k)}:=\left(\sum_{m \in I_{2}} \tilde{g}^{\{m\}, m}\right) \leftarrow\left(x^{(j, k)}\right), j \in\{1, \ldots, k-1\},
$$

where $\leftarrow$ denotes the generalized inverse for non-increasing functions $s^{6}$ and for $k \in\{0, \ldots, k\}$

$$
x^{(j, k)}:=\frac{k-j}{k} \sum_{m \in I_{2}} \tilde{g}^{\{m\}, m}\left(t_{0}\right)+\frac{j}{k} \sum_{m \in I_{2}} \tilde{g}^{\{m\}, m}\left(s_{0}\right) .
$$

As $\tilde{g}^{\{m\}, m}$ are continuous and non-decreasing the generalized inverse is a right-inverse 7 and

$$
\begin{aligned}
\mu_{I_{2}}\left(t^{(j, k)}, t^{(j-1, k)}\right) & =\underbrace{\sum_{m \in I_{2}} \tilde{g}^{\{m\}, m}\left(t^{(j-1, k)}\right)}_{=x^{(j-1, k)}}-\underbrace{\sum_{m \in I_{2}} \tilde{g}^{\{m\}, m}\left(t^{(j, k)}\right)}_{=x^{(j, k)}} \\
& =\frac{1}{k} \mu_{I_{2}}\left(s_{0}, t_{0}\right) .
\end{aligned}
$$

${ }^{6}$ For a non-increasing function $f$, its generalized inverse is defined by $f^{\leftarrow}(x):=\inf \{x$ : $f(x) \leqslant y\}$ and for a non-decreasing function $f$, its generalized inverse is defined by $f^{\leftarrow}(x):=$ $\inf \{y: f(y) \geqslant x\}$.

7 If $g$ is a continuous and non-increasing function, then $g^{\leftarrow}(x)=(-g)^{\leftarrow}(-x)$, where the generalized inverse on the l.h.s. is for non-increasing and on the r.h.s. for non-decreasing functions. As $(-g)^{\leftarrow}$ is a right-inverse of $-g$, see [6. p.425 sq., prop. 1 (4)], this implies that $\mathrm{g} \leftarrow$ is a right-inverse of $\mathrm{g}$. 
Assume that for all $j \in\{1, \ldots, k\}$ the following inequality holds

$$
\bar{S}_{I_{1}, I_{2}}\left(t^{(j-1, k)}\right)-\bar{S}_{I_{1}, I_{2}}\left(t^{(j, k)}\right)>-a\left(s_{0}, t_{0}\right) \mu_{I_{2}}\left(t^{(j, k)}, t^{(j-1, k)}\right) .
$$

Then,

$$
\begin{aligned}
& \bar{S}_{I_{1}, I_{2}}\left(t_{0}\right)-\bar{S}_{I_{1}, I_{2}}\left(s_{0}\right) \\
& =\sum_{j=1}^{k} \bar{S}_{I_{1}, I_{2}}\left(t^{(j-1, k)}\right)-\bar{S}_{I_{1}, I_{2}}\left(t^{(j, k)}\right) \\
& >-a\left(s_{0}, t_{0}\right) \sum_{j=1}^{k} \mu_{I_{2}}\left(t^{(j, k)}, t^{(j-1, k)}\right) \\
& =-a\left(s_{0}, t_{0}\right) \mu_{I_{2}}\left(s_{0}, t_{0}\right),
\end{aligned}
$$

which is a contradiction. Hence, with $t_{k}=t^{(j-1, k)}, s_{k}=t^{(j, k)}$ for some $j \in\{1, \ldots, k\}$, Eq. 18$)$ is fulfilled and $s_{k}>t_{k}$.

Combining Eq. (17) with these results gives for feasible $t_{k}, s_{k}$ (chosen as above)

$$
\begin{aligned}
& 0 \leqslant G_{I_{1}, I_{2}}^{\left\{g_{\left|I_{1}\right|+1}^{\pi_{J}}, g_{\left|I_{1}\right|+2}^{\pi_{J}}, \ldots, g_{\left|I_{1} \cup I_{2}\right|}^{\pi_{J}}\right\}_{J \in I_{2}}}\left(s_{k}, t_{k}\right) \\
& =\underbrace{\hat{g}_{\left|I_{1}\right|+1}^{\pi_{\emptyset}}\left(t_{k}\right)}_{\pi_{\emptyset}} g_{\left|I_{1}\right|+2}^{\pi_{\emptyset}}\left(t_{k}\right) \cdot \ldots \cdot g_{\left|I_{1} \cup I_{2}\right|}^{\pi_{\emptyset}}\left(t_{k}\right) \\
& =\frac{g_{\left|I_{1}\right|+1}^{\pi_{\emptyset}}\left(t_{k}\right)}{\bar{S}_{I_{1}, I_{2}}\left(t_{k}\right)} \\
& \times \underbrace{\left(\bar{S}_{I_{1}, I_{2}}\left(t_{k}\right)-\bar{S}_{I_{1}, I_{2}}\left(s_{k}\right)\right)}_{\leqslant-a\left(s_{0}, t_{0}\right) \frac{\mu_{I_{2}}\left(s_{0}, t_{0}\right)}{k}} \\
& +\bar{S}_{\mathrm{I}_{1}, \mathrm{I}_{2}}\left(s_{\mathrm{k}}\right) \mathrm{G}_{\mathrm{I}_{1}, \mathrm{I}_{2}}\left\{\hat{\mathrm{g}}_{\mathrm{I}_{\mathrm{J}} \mid+1}^{\left.\pi_{\mathrm{N}}, \mathrm{g}_{\left|\mathrm{I}_{1}\right|+2}, \ldots, \mathrm{g}_{\left|\mathrm{I}_{1} \cup \mathrm{I}_{2}\right|}^{\pi_{\mathrm{J}}}\right\}_{\mathrm{J} \in \mathrm{I}_{2}}}\left(s_{\mathrm{k}}, \mathrm{t}_{\mathrm{k}}\right)\right. \\
& \leqslant \frac{g_{\left|\mathrm{I}_{1}\right|+1}^{\pi_{\emptyset}}\left(s_{0}\right)}{p_{\max }^{\mathrm{I}_{1}, \mathrm{I}_{2}}\left(s_{0}, \mathrm{t}_{0}\right)} g_{\left|\mathrm{I}_{1}\right|+2}^{\pi_{\emptyset}}\left(s_{0}\right) \cdot \ldots \cdot g_{\left|\mathrm{I}_{1} \cup \mathrm{I}_{2}\right|}^{\pi_{\emptyset}}\left(s_{0}\right) \\
& \times\left(-a\left(s_{0}, t_{0}\right) \mu_{I_{2}}\left(s_{0}, t_{0}\right) \frac{1}{k}\right) \\
& +b p_{\max }^{I_{1}, I_{2}}\left(s_{0}, t_{0}\right) p_{I_{1}, I_{2}}\left(s_{0}, t_{0}\right) \mu_{I_{2}}\left(s_{0}, t_{0}\right)^{2} \frac{1}{k^{2}} \text {. }
\end{aligned}
$$

In particular, if the latter inequality is multiplied by $k$ and the limit $k \rightarrow \infty$ 
is taken, then

$$
0 \leqslant-\underbrace{\frac{1}{p_{\text {max }}^{I_{1}, I_{2}}\left(s_{0}, t_{0}\right)}}_{>0} \underbrace{a\left(s_{0}, t_{0}\right)}_{>0} \underbrace{\mu_{I_{1}, I_{2}}\left(s_{0}, t_{0}\right)}_{>0} \underbrace{\prod_{j=1}^{\left|I_{2}\right|} g_{\left|I_{1}\right|+j}^{\pi_{\emptyset}}\left(s_{0}\right)}_{>0}<0,
$$

which leads to a contradiction.

\section{Applications and Outlook}

An additive subordinator is a stochastic process $\Lambda=\{\Lambda(t)\}_{t} \geqslant 0$ on the nonnegative real line $[0, \infty]$, which starts at zero, is stochastically continuous, càdlàg, and has independent increments. Note that this implies that $\Lambda$ has a.s. non-decreasing path. It can be shown, see [17], that the distribution of an additive subordinator $\Lambda$ can uniquely be identified with a family of Bernstein functions $8\left\{\left\{\psi_{t}(x)\right\}_{t} \geqslant 0\right.$ via $\psi_{t}(x)=-\log \mathbb{E}[\exp \{-x \Lambda(t)\}]$ and it holds that

(1) $\psi_{0}(x)=\delta_{0}(x)$, where $\delta_{0}$ is the Dirac-measure in zero,

(2) $x \mapsto\left(\psi_{s}(x)-\psi_{t}(x)\right)$ is a Bernstein function for all $s>t \geqslant 0$,

(3) $t \mapsto \psi_{t}(x)$ is continuous for all $x \geqslant 0$.

It was shown in [17] that the random vector $\tau$ belongs to the class of exchangeable generalized Marshall-Olkin distributions which have a stochastic representation as an exchangeable exogenous shock model, where

$$
\tau_{i}:=\left\{t>0: \Lambda_{i}(t)>E_{i}\right\}, \quad i \in[d],
$$

$\Lambda_{i} \equiv \Lambda$ is an additive subordinator, and $\left\{E_{i}\right\}_{i \in[d]}$ are iid unit exponential random variables independent of $\Lambda$. Furthmore, if $\psi_{t}(x)=-\log \mathbb{E}[\exp \{-x \wedge(t)\}]$, it holds for $t \geqslant 0$ and $\pi \in \mathcal{S}_{\mathrm{d}}$ with $t_{\pi(1)} \geqslant \ldots \geqslant t_{\pi(d)}$ that

$$
\mathbb{P}(\boldsymbol{\tau}>\mathbf{t})=\prod_{i=1}^{\mathrm{d}} \exp \left\{-\left(\psi_{\mathrm{t}_{\pi(i)}}(\mathfrak{i})-\psi_{\mathrm{t}_{\pi(i)}}(i-1)\right)\right\} .
$$

\footnotetext{
${ }^{8} \mathrm{~A}$ Bernstein function is a non-negative, infinitely often differentiable function $\psi:[0, \infty) \rightarrow$ $[0, \infty)$ with $(-1)^{n+1} \psi^{(n)} \geqslant 0$. Standard literature, see, e.g., $|1,23|$, states that the class of Bernstein functions is represented as $\left\{x \mapsto a 1_{(0, \infty)}(x)+b x+\int_{0, \infty}(1-\exp \{-x s\}) v(d s)\right.$ : $a, b \geqslant 0, v$ is a Lévy-measure\}.
} 
This model is called exchangeable additive-frailty model (exAFM) and Thm. 国, or its exchangeable version in [17], implies that $\tau$ has an alternative representation as an exchangeable exogenous shock model. The exAFM can be generalized to produce non-exchangeable random vectors as the following factor model construction shows: Assume that $\tau$ is defined by Eq. (19), where $\Lambda_{i}$ are additive subordinators from the convex cone which is spanned by independent additive subordinators $\Upsilon^{(1)}, \ldots, \Upsilon^{(n)}$ (independent of $E_{1}, \ldots, E_{d}$ ), i.e.

$$
\Lambda_{i}(t)=\theta_{i}^{\prime} \Upsilon, \quad i \in[d],
$$

for some $n \in \mathbb{N}$ and $\theta_{i} \in[0, \infty)^{n} \backslash\{0\}, i \in[d]$. A straightforward calculation, similar to the one in [17. Prop. 3.1], shows that for $\psi_{t}^{(k)}(x)=$ $-\log \mathbb{E}\left[\exp \left\{-x \Upsilon^{(k)}(t)\right\}\right], t \geqslant 0$, and $\pi \in \mathcal{S}_{d}$ with $t_{\pi(1)} \geqslant \ldots \geqslant t_{\pi(d)}$

$$
\begin{array}{r}
\mathbb{P}(\boldsymbol{\tau}>\mathbf{t})=\prod_{i=1}^{\mathrm{d}} \prod_{k=1}^{n} \exp \left\{-\left[\psi_{\mathrm{t}_{\pi(i)}}^{(k)}\left(\sum_{j=1}^{i} \Theta_{\pi(i), k}\right)\right.\right. \\
\left.\left.-\psi_{\mathrm{t}_{\pi(i)}}^{(k)}\left(\sum_{j=1}^{i-1} \Theta_{\pi(i), k}\right)\right]\right\},
\end{array}
$$

where $\Theta=\left(\theta_{1}, \ldots, \theta_{n}\right)^{\prime}$.

This model can be used to define hierarchical models similar to those introduced in [16]. It follows with Thm. 1 that $\tau$ has a generalized MarshallOlkin distribution, i.e. it has an alternative stochastic representation as an exogenous shock model and the shock distributions can be calculated from the Bernstein functions using the discrete difference operator: Let $s>t \geqslant 0$ and $\emptyset \neq I \subseteq[d]$ with $I=\left\{i_{1}, \ldots, i_{|I|}\right\}$; then the shock survival function $\bar{H}_{I}$ fulfills

$$
\begin{aligned}
\frac{\overline{\mathrm{H}}_{\mathrm{I}}(\mathrm{s})}{\overline{\mathrm{H}}_{\mathrm{I}}(\mathrm{t})}=\exp \left\{(-1)^{|\mathrm{I}|} \sum_{\mathrm{k}=1}^{n} \Delta_{\Theta_{\mathrm{i}_{|I|}, k}} \ldots \Delta_{\Theta_{i_{1}, k}}\right. \\
\left.\quad\left(\psi_{\mathrm{s}}^{(\mathrm{k})}-\psi_{\mathrm{t}}^{(\mathrm{k})}\right)\left(\sum_{j \in[\mathrm{d}] \backslash \mathrm{I}} \Theta_{j, k}\right)\right\} .
\end{aligned}
$$

This connection between the (hierarchical) additive-frailty model and exogenous shock models can be used in multiple ways, e.g., as shown in the following to calculate joint failure probabilities via numerical integration: 
Let $(t, x) \mapsto \psi_{t}^{(k)}(x)$ differentiable w.r.t. $t$ and their partial derivatives w.r.t. $t$ be continuous in $x$ and $t$. Then

$$
\begin{aligned}
\mathbb{P}\left(\tau_{1}=\ldots=\tau_{\mathrm{d}}\right)=\mathbb{P}\left(\mathrm{Z}_{[\mathrm{d}]}<\min _{\emptyset \neq \mathrm{I} \subsetneq[\mathrm{d}]} \mathrm{Z}_{\mathrm{I}}\right) \\
=\mathbb{E}\left[\mathbb{P}\left(\mathrm{Z}_{[\mathrm{d}]}<\min _{\emptyset \neq \mathrm{I} \subsetneq[\mathrm{d}]} \mathrm{Z}_{\mathrm{I}} \mid \mathrm{Z}_{[\mathrm{d}]}\right)\right]=\int_{0}^{\infty} \overline{\mathrm{F}}(z) \cdot \frac{-\frac{\partial}{\partial z} \overline{\mathrm{H}}_{[\mathrm{d}]}(z)}{\overline{\mathrm{H}}_{[\mathrm{d}]}(z)} \mathrm{d} z \\
=\int_{0}^{\infty} \exp \left\{-\sum_{k=1}^{n} \psi_{z}^{(\mathrm{k})}\left(\sum_{j=1}^{\mathrm{d}} \Theta_{j \mathrm{k}}\right)\right\} \\
\times\left[(-1)^{\mathrm{d}+1} \frac{\partial}{\partial z} \sum_{\mathrm{k}=1}^{n} \Delta_{\Theta_{1, k}} \ldots \Delta_{\Theta_{\mathrm{d}, \mathrm{k}}} \psi_{z}^{(\mathrm{k})}(0)\right] \mathrm{d} z
\end{aligned}
$$

where $\left\{Z_{\mathrm{I}}: \emptyset \neq i \subseteq[\mathrm{d}]\right\}$ are independent shocks of a corresponding exogenous shock model and the last step follows with Eqs. 21) and 22. One can also use integration by parts to show that

$$
\begin{aligned}
& \mathbb{P}\left(\tau_{1}=\ldots=\tau_{d}\right) \\
& =\underbrace{\left.\overline{\mathrm{F}}(z) \cdot\left[-\log \mathrm{H}_{[\mathrm{d}]}(z)\right]\right|_{0} ^{\infty}}_{\stackrel{(\star)}{=} 0}+\int_{0}^{\infty}\left[\frac{\partial}{\partial z} \overline{\mathrm{F}}(z)\right] \cdot \log \overline{\mathrm{H}}_{[\mathrm{d}]}(z) \mathrm{d} z \\
& =\int_{0}^{\infty}\left[\frac{\partial}{\partial z} \sum_{k=1}^{n} \psi_{z}^{(k)}\left(\sum_{j=1}^{\mathrm{d}} \Theta_{j k}\right)\right] \\
& \times \exp \left\{-\sum_{k=1}^{n} \psi_{z}^{(k)}\left(\sum_{j=1}^{d} \Theta_{j k}\right)\right\} \\
& \times\left[(-1)^{\mathrm{d}+1} \sum_{\mathrm{k}=1}^{\mathrm{n}} \Delta_{\Theta_{1, \mathrm{k}}} \ldots \Delta_{\Theta_{\mathrm{d}, \mathrm{k}}} \psi_{z}^{(\mathrm{k})}(0)\right] \mathrm{d} z,
\end{aligned}
$$

where $(\star)$ follows with $\lim _{x \rightarrow \infty} x e^{-x}=0$ and from Eqs. 21 and 22, as well 
as the Bernstein property of the functions $\psi^{(k)}$, as these imply for $k \in[n]$

$$
\begin{aligned}
& {\left[(-1)^{\mathrm{d}+1} \Delta_{\Theta_{1, k}} \ldots \Delta_{\Theta_{\mathrm{d}, \mathrm{k}}} \psi_{z}(0)\right]} \\
& \quad=\underbrace{(-1)^{\mathrm{d}+1} \Delta_{\Theta_{1, k}} \ldots \Delta_{\Theta_{\mathrm{d}-1, \mathrm{k}}} \psi_{z}\left(\Theta_{\mathrm{d}, \mathrm{k}}\right)}_{\leqslant 0}+(-1)^{\mathrm{d}} \Delta_{\Theta_{1, \mathrm{k}}} \ldots \Delta_{\Theta_{\mathrm{d}-1, \mathrm{k}}} \psi_{z}(0) \\
& \quad \leqslant(-1)^{\mathrm{d}} \Delta_{\Theta_{1, \mathrm{k}}} \ldots \Delta_{\Theta_{\mathrm{d}-1, \mathrm{k}}} \psi_{z}(0) \leqslant \ldots \leqslant \Delta_{\Theta_{1, k}} \psi_{z}(0) \leqslant \psi_{z}\left(\Theta_{1, \mathrm{k}}\right) \\
& \quad \leqslant \psi_{z}\left(\sum_{j=1}^{\mathrm{d}} \Theta_{j, \mathrm{k}}\right) .
\end{aligned}
$$

Note that in case the underlying model is exchangeable with $\psi=\psi_{1}^{(1)}$ and $\Delta=\Delta_{1}$, then

$$
\begin{aligned}
\mathbb{P}\left(\tau_{1}=\ldots=\tau_{\mathrm{d}}\right) & \stackrel{\text { Eq. 23) }}{=} \int_{0}^{\infty} \mathrm{e}^{-z \psi(\mathrm{d})} \cdot\left[(-1)^{\mathrm{d}+1} \Delta^{\mathrm{d}} \psi(0)\right] \mathrm{d} z \\
& =\frac{(-1)^{\mathrm{d}+1} \Delta^{\mathrm{d}} \psi(0)}{\psi(\mathrm{d})}=\frac{\sum_{i=0}^{\mathrm{d}}\left(\begin{array}{l}
\mathrm{d} \\
i
\end{array}\right)(-1)^{\mathrm{i}+1} \psi(\mathrm{i})}{\psi(\mathrm{d})} .
\end{aligned}
$$

Equations (21) and (22) have been tested with a simple implementation for the case that $n=1, \Theta=1$, and $\psi=\psi^{(1)}$ is the Bernstein function of a compound Poisson subordinator with exponentially distributed jumps, i.e. $\psi_{t}(x)=\mu x t+\beta t \cdot(1-\eta /(x+\eta))$ for $(\mu, \beta, \eta) \geqslant 0$, where exact formulas of the "combined death"-probability are known, see [15, p. 111 sq.]. The three parameter combinations from [18, Fig. 3.6, p.156 sq. $]^{5}$ were used and showed similar results: The exact formula as well as the formula from Eq. 24) perform equally well up to $d \approx 50$ and the formula from Eq. 23) performs well up to $d \approx 25$. The breakdown, which can be detected using the monotonicity properties of the Bernstein function $\psi$, is due to loss of significant digits in the numerical calculation of the discrete differences. Moreover, for small $\mathrm{d}$ the numerical integration formula outperforms a Monte-Carlo estimation of the probabilities w.r.t. error-size as well as runtime.

In case that $n=1$ and $\Theta=1$, i.e. if the model is exchangeable, and $\Lambda=\Upsilon^{(1)}$ is a Lévy subordinator, the model can be (uniquely) linked to so called regenerative composition structures, see [8], In that case, the

\footnotetext{
${ }^{9}$ These are $(0.2995,1.401,1),(0.2,2.4,2)$, and $(0.0151,0.994749,0.01)$.

${ }^{10}$ For a definition of (regenerative) composition structures and an introduction of the notation which is used hereinafter, the interested reader is referred to [8].
} 
corresponding shock model is a classical Marshall-Olkin model and the decrement matrix of the corresponding regenerative composition model can be expressed in terms of the exponential rates of the exchangeable MO-distribution $\left\{\lambda_{\mathfrak{m}}^{(\mathfrak{n})}, 1 \leqslant m \leqslant n\right\}$, i.e.

$$
\begin{aligned}
\mathrm{q}(\mathrm{n}: \mathrm{m}) & =\mathbb{P}\left(\min _{\emptyset \neq \mathrm{I} \subseteq[\mathrm{d}]:|\mathrm{I}|=\mathrm{m}} \mathrm{Z}_{\mathrm{I}}^{(\mathrm{n})}<\min _{\emptyset \neq \mathrm{I} \subseteq[\mathrm{d}]:|\mathrm{I}| \neq m} \mathrm{Z}_{\mathrm{I}}^{(\mathrm{n})}\right) \\
& =\frac{\left(\begin{array}{c}
n \\
m
\end{array}\right) \lambda_{m}^{(n)}}{\sum_{k=1}^{n}\left(\begin{array}{l}
n \\
k
\end{array}\right) \lambda_{k}^{(n)}},
\end{aligned}
$$

where $\left\{Z_{I}^{(\mathfrak{n})}\right\}_{\emptyset \neq I \subseteq[\mathrm{d}]}$ are independend exponential random variables with rates $\lambda_{\mathrm{I}}^{(\mathrm{n})} \equiv \lambda_{|\mathrm{I}|}^{(\mathrm{n})}$ and

$$
\lambda_{m}^{(n)}=\sum_{j=0}^{m}(-1)^{j+1}\left(\begin{array}{c}
m \\
j
\end{array}\right) \psi(n-m+j), \quad 1 \leqslant m \leqslant n .
$$

Thm. 1 can subsequently be used to extend some results from [8] for composition structures which fulfill a suitably relaxed notion of regenerativity such that the stochastic process representation uses an additive subordinator instead of a Lévy subordinator.

\section{Conclusion}

The survival functions of ESM distributions are the product of their ordered and individually transformed arguments. The transformations $g_{i}^{\pi}$ are orderdependent if the ESM distribution is not exchangeable. Conversely, if a function of that form is a continuous multivariate survival function, the distribution has a stochastic representation as an exogenous shock model. Formulas for retrieving the shock survival functions from the transformations $g_{i}^{\pi}$ are given explicitly. Furthermore, the special form of $\bar{F}(t)=\prod_{i=1}^{d} g_{i}^{\pi}\left(t_{\pi(i)}\right)$ implies a simplified d-volume condition. The attained results generalize the findings from $|17|$ for the exchangeable subclass.

\section{References}

[1] Christian Berg, Jens Peter Reus Christensen, and Paul Ressel. Harmonic analysis on semigroups. Vol. 100. Graduate Texts in Mathematics. 


\section{[2]} der Herbertsson. "A bottom-up dynamic model of portfolio credit risk: part ii: common-shock interpretation, calibration and hedging issues". Available at SSRN: https://ssrn.com/abstract $=2245130$ or http:/ /dx.doi.org/10.2139/ssrn.2245130. Mar. 2013.

[3] Fabrizio Durante, Anna Kolesárová, Radko Mesiar, and Carlo Sempi. "Semilinear Copulas". In: Fuzzy Sets and Systems 159.1 (2008), pp. 63-76. DOI: 10.1016/j.fss.2007.09.001.

[4] Fabrizio Durante, José Juan Quesada-Molina, and Manuel ÚbedaFlores. "On a family of multivariate copulas for aggregation processes". In: Information Sciences 177.24 (2007), pp. 5715-5724. DOI: 10.1016/j.ins.2007.07.019.

[5] Youssef Elouerkhaoui. "Pricing and Hedging in a Dynamic Credit Model". In: International Journal of Theoretical and Applied Finance 10.04 (2007), pp. 703-731. DOI: 10.1142/S0219024907004408.

[6] Paul Embrechts and Marius Hofert. "A note on generalized inverses". In: Mathematical Methods of Operations Research 77.3 (2013), pp. 423-432.

[7] Kay Giesecke. "A simple exponential model for dependent defaults". In: The Journal of Fixed Income 13.3 (2003), pp. 74-83. Dor: 10.3905/jii. 2003.319362.

[8] Alexander Gnedin and Jim Pitman. "Regenerative composition structures". In: Ann. Probab. 33.2 (Mar. 2005), pp. 445-479. Dor: 10.1214/ 009117904000000801, uRL: https://doi.org/10.1214/009117904000000801.

[9] Harry Joe. Multivariate models and multivariate dependence concepts. CRC Press, 1997.

[10] John P. Klein, Niels Keiding, and Claus Kamby. "Semiparametric Marshall-Olkin Models Applied to the Occurrence of Metastases at Multiple Sites after Breast Cancer". In: Biometrics 45.4 (1989), pp. 10731086.

[11] Haijun Li. "Orthant tail dependence of multivariate extreme value distributions". In: Journal of Multivariate Analysis 100.1 (2009), pp. 243256. DOI: https://doi.org/10.1016/j.jmva.2008.04.007. 
[12] Xiaohu Li and Franco Pellerey. "Generalized Marshall-Olkin distributions and related bivariate aging properties". In: Journal of Multivariate Analysis 102.10 (2011), pp. 1399-1409. Dor: http://dx.doi.org/10.1016/ j.jmva.2011.05.006.

[13] Jianhua Lin and Xiaohu Li. "Multivariate Generalized Marshall-Olkin Distributions and Copulas". In: Methodology and Computing in Applied Probability 16.1 (2014), pp. 53-78. Dor: 10.1007/s11009-012-9297-4

[14] Filip Lindskog and Alexander J. McNeil. "Common Poisson shock models: applications to insurance and credit risk modelling". In: Astin Bulletin 33.2 (2003), pp. 209-238. Dor: 10.1017/S0515036100013441.

[15] Jan-Frederik Mai. "Extendibility of Marshall-Olkin distributions via Lévy subordinators and an application to portfolio credit risk". Available at http:/ / mediatum.ub.tum.de?id=969547. Dissertation. Technische Universität München, 2010.

[16] Jan-Frederik Mai. "Multivariate exponential distributions with latent factor structure and related topics". Habilitation thesis. Technische Universität München, 2014.

[17] Jan-Frederik Mai, Steffen Schenk, and Matthias Scherer. "Exchangeable exogenous shock models". In: Bernoulli 22.2 (2016), pp. 1278-1299. DoI: 10.3150/14-BEJ693.

[18] Jan-Frederik Mai and Matthias Scherer. Simulating copulas: stochastic models, sampling algorithms and applications. 2nd ed. Vol. 6. Series in Quantitative Finance. World Scientific, 2017. ISBN: 978-981-3149-24-3.

[19] Albert W. Marshall. "Copulas, marginals, and joint distributions". In: Distributions with fixed marginals and related topics. Ed. by Ludger Rüschendorf, Berthold Schweizer, and Michael D. Taylor. Vol. Volume 28. Lecture Notes-Monograph Series. Institute of Mathematical Statistics, 1996, pp. 213-222. DOI: $10.1214 / \mathrm{lnms} / 1215452620$.

[20] Albert W. Marshall and Ingram Olkin. "A multivariate exponential distribution". In: Journal of the American Statistical Association 62.317 (1967), pp. 30-44. DOr: 10.2307/2282907.

[21] Pietro Muliere and Marco Scarsini. "Characterization of a MarshallOlkin type class of distributions". In: Annals of the Institute of Statistical Mathematics 39.1 (1987), pp. 429-441. DoI: 10.1007/BF02491480. 
[22] Steffen Schenk. "Exchangeable Exogenous Shock Models". Dissertation. Munich: Technical University of Munich, 2016.

[23] René L Schilling, Renming Song, and Zoran Vondracek. Bernstein functions. 2nd rev. and ext. ed. Vol. 37. De Gruyter Studies in Mathematics. Berlin: De Gruyter, 2012. ISBN: 978-3-11-026933-8.

[24] B. Schweizer and A. Sklar. Probabilistic metric spaces. Elsevier/NorthHolland, 1983.

[25] Abe Sklar. "Fonctions de répartition a n dimensions et leurs marges". In: Publ. Inst. Statist. Univ. Paris 8 (1959), pp. 229-231. 